\title{
Linguistic landscapes in der Innerschweiz: Dialekt, Toponyme und he- raldische Zeichen als Ausdruck von Raumzugehörigkeit*
}

\author{
Marina Petkova (Freiburg i. Ü.)
}

\begin{abstract}
This article explores the linguistic landscapes of four towns in Central Switzerland. The analysis focuses on linguistic features such as words written in dialect or toponyms and on some features from other semiotic codes like heraldic signs or arrangements in shop windows which include language, pictures, flags etc. The main interest lies in the way the mental concepts of "space" and "place" are displayed in the linguistic landscape. Which geographical categories are relevant? Which features tend to express a sense of belonging? The analysis shows that the linguistic landscape of the four towns contains features that represent several different mental concepts of "space" and "place". The most significant ones are those that represent the close environment, i. e. the part of the world that tends to be felt as being a "place". Thus, the linguistic landscape also reflects the effects of "place-making"-activities and at the same time visualizes the relationship between physical localities, mental concepts of "space" or "place" and human activities.
\end{abstract}

\section{$1 \quad$ Einleitung}

In diesem Beitrag wird der Ausdruck von Zugehörigkeit zu bestimmten Räumen thematisiert. Die Basis bildet die linguistic landscapes-Forschung, eine jüngere Forschungsrichtung innerhalb der Linguistik, die sich mit der ,ding- und ortfesten Schriftverwendung im öffentlichen Raum“ (Auer 2010: 273) beschäftigt. Solche sprachlichen Zeichen prägen nicht nur das Erscheinungsbild einer Ortschaft, sondern sagen einiges über die sozialen Verhältnisse und über die (Selbst)Positionierung der dort lebenden Menschen aus. Um das Datenmaterial der hier vorgestellten Studie zu interpretieren, werden einige Überlegungen zum Verhältnis zwischen Ort, Raum und Sprache der Analyse vorangestellt. Wichtig dabei ist die Auffassung von Raum als konzeptuelle Grösse, die zwar auf physisch-materielle Gegebenheiten Bezug nimmt, doch ebenso mit der Vorstellungswelt und dem Wissen des Individuums verbunden ist und das raumbezogene (sprachliche) Handeln beeinflusst. Die Ausprägung der linguistic landscape ist als Resultat eines solchen raumbezogenen sprachlichen Handelns zu betrachten und kann Aufschluss über die dahinterstehenden Raumkonzepte geben. Im Zentrum des Inte-

\footnotetext{
* Der Anstoss zu dieser kleinen Studie kam von Christina Ada Anders, wofür ihr an dieser Stelle herzlich gedankt sei. Mein Dank gilt auch Evelyn Ziegler für wertvolle Impulse im Zusammenhang mit der Materialauswertung. Helen Christen, Ingrid Hove und Alexandra Schiesser danke ich für die hilfreichen Kommentare zum Text. Linguistik online 85, 6/17 - http://dx.doi.org/10.13092/lo.85.4085
}

CC by 3.0 
resses stehen vier Gemeinden in der Innerschweiz (Altdorf, Schwyz, Sarnen und Stans), die sowohl bezüglich ihres Territoriums als auch bezüglich der Bevölkerungszahl klein sind, dabei aber gleichzeitig als Kantonshauptorte fungieren. Somit wird das Interesse auf kleinere geographische Grössen gelenkt, wie sie bisher weniger im Fokus der linguistic landscapeForschung gestanden sind. Die zentralen Fragen sind: Finden sich in der linguistic landscape dieser Ortschaften Raumbezüge? Welche geographischen Grössen kommen vor? Welche Mittel bringen das Zugehörigkeitsempfinden der Menschen zum Ausdruck?

\section{Raum als mentale Konstruktion}

Die Konzeptualisierung des Raumes als eine Art Behälter oder Container, der Objekte und Menschen einschliesst, war längere Zeit vorherrschend im westlichen Denken (Löw 2012: Kap. 2; Auer 2013: 5). Die Geographie als diejenige Wissenschaftsdisziplin, die sich primär mit dem Raum beschäftigt, blickt auf eine Geschichte zurück, an deren Ausgangspunkt die Bemühung stand, die physische Welt zu vermessen und zu beschreiben. Dabei etablierte sich zunächst eine Sicht, wonach die Naturgegebenheiten für Gesellschaft und Kultur prägend seien. Die weitere Entwicklung erfolgt über einen immer grösseren Miteinbezug der Wechselwirkungen zwischen Mensch und Umwelt. Die Effekte des menschlichen Handelns und die Art, wie sich dieses auf die Landschaft auswirkt bzw. die Kräfte und Prozesse, die dahinterstehen, bilden vermehrt einen Interessenschwerpunkt. Die Humangeographie stellt schliesslich den Menschen gänzlich ins Zentrum. Sie interessiert sich dafür, wie dieser seine Umwelt erlebt und betrachtet die Zusammenhänge zwischen Raum und menschlicher Erfahrung aus der Perspektive des Individuums (zu diesen Entwicklungen cf. Cresswell 2004: Kap. 2, Johnstone 2010). Aber auch andere Wissenschaftsdisziplinen wie die Soziologie oder die Linguistik haben sich mittlerweile von der Vorstellung gelöst, wonach der physisch-materielle geographische Raum als eine für sich existierende Grösse bereits das Vorkommen bestimmter Sprachen oder sprachlicher Merkmale determiniert (cf. hierzu die Kritik in Auer 2004). Es setzt sich vielmehr eine Sicht durch, wonach „Raum“ als mentale Konstruktion zu betrachten ist, denn die "physical aspects [...] are always mediated by subjective experience" (Johnstone 2010: 10). Nicht minder wichtig ist dabei die Handlungskomponente. Aus soziologischer Sicht schreibt Löw (2012: 204) hierzu: „Räume werden im Handeln geschaffen, indem Objekte und Menschen synthetisiert und relational angeordnet werden." 1

Dass der „Raum“ als Konzept viel mehr beinhaltet als die physisch-materiellen Gegebenheiten, ist mittlerweile eine akzeptierte und verbreitete Sicht. Die theoretische Umsetzung des Verhältnisses zwischen der materiellen und der mentalen Komponente wird jedoch unterschiedlich gehandhabt. So kann von einer besonders menschenzentrierten Sicht aus gefragt werden, "whether it is possible to speak of space independent of human activity" (Johnstone 2010: 8). Humangeographen wie J. Nicholas Entrikin vertreten eine Zwischenposition und sprechen von der "'betweenness" of place, partly physical, but always also partly the result of social activity" (Johnstone 2010: 8). Hinzu kommt eine begriffliche Unterscheidung, die in der englischsprachigen Diskussion das Verhältnis zwischen space und place, in der deutsch-

\footnotetext{
${ }^{1}$ Synthese und relationale Anordnung sind hier mentale Prozesse, die zur Konstruktion von „Raum“ als mentale Grösse führen.
} 
sprachigen Diskussion dasjenige zwischen „Raum“ und „Ort“ betrifft. Wie die Begriffe zueinander positioniert werden, unterscheidet sich von Forscherposition zu Forscherposition wesentlich, was die Auseinandersetzung mit der Forschungsliteratur zuweilen schwierig macht. "In general, space is seen as the raw material for the construction, by human societies, of landscape, or, more abstractly, place”, schreibt Johnstone (2010: 8). Ob nun dieses „Rohmaterial“" die physisch-materielle Komponente einschliesst oder ebenfalls auf einer abstrakten Ebene zu finden ist, bleibt indes unklar. Einige der in Johnstone (2010) zitierten und diskutierten Forscher verwenden "space" zur Bezeichnung sowohl der materiellen als auch der mentalen Komponente. Dies ist der Fall bei Soja (1989: 120, zit. nach Johnstone 2010: 8), der zwischen einem "physical space of material nature" und einem "mental space of cognition and representation" unterscheidet, und kommt auch in der Aussage von Bridge und Watson (2000: 7, zit. nach Johnstone 2010: 11) zum Vorschein, wonach "cities are not simply material or lived spaces - they are also spaces of the imagination and spaces of representation". Für andere bezieht sich der Begriff erst auf die kulturell überformte Ausprägung der natürlichen Gegebenheiten, er erhält also eine ausgeprägte soziale Dimension (so z. B. bei Lefebvre 1980, aus dessen Sicht « espace » explizit vom Menschen produziert wird).

Eine ganz andere Sicht wird in der aktuellen Diskussion vertreten, bei der das Konzept place im Mittelpunkt steht (cf. den ausführlichen Literaturüberblick in Gieryn 2000 aus soziologischer und in Cresswell 2004 aus humangeographischer Sicht). Hier wird space als die abstraktere, inhalts- und bedeutungsärmere Komponente innerhalb einer häufig dichotomischen Gegenüberstellung von space und place betrachtet. Der Begriff place bleibt Räumen vorbehalten, mit denen die Menschen eine emotionale Verbindung aufgebaut haben, die mit Bedeutung angereichert sind. Cresswell schreibt hierzu:

Space, then, has been seen in distinction to place as a realm without meaning - as a 'fact of life' which, like time, produces the basic coordinates for human life. When humans invest meaning in a portion of space and then become attached to it in some way (naming is one such way) it becomes a place.

(Cresswell 2004: 10)

Für Gieryn (2000: 465) ist space "what place becomes when the unique gathering of things, meanings, and values are sucked out [...]. Put positively, place is space filled up by people, practices, objects, and representations". In Bezug auf die Materialität von place beziehen die beiden Überblicksdarstellungen dennoch deutlich unterschiedliche Positionen. Während für Gieryn (2000: 465) place "physicality" hat, ist dieses für Cresswell (2004: 33) "a construction of humanity". Die Schaffung von place als mehr oder weniger bewusste Aktivität der Menschen stellt wiederum in beiden Forschungslinien einen unbestrittenen Schwerpunkt dar. Ein Zustand der Abgeschlossenheit kann dabei nicht erreicht werden. "In general places are never complete, finished or bounded but are always becoming - in process", schreibt Cresswell (2004: 37). Die dahinterstehenden Prozesse, "by which anonymous space is turned into "somebody's' place" (Auer 2013: 16), werden als "place-making” bezeichnet. In diesem Sinne sollte das Verhältnis zwischen space und place, wenngleich häufig als dichotomisch erfasst, eher als ein Kontinuum begriffen werden. Eine solche Sicht scheint gemäss Cresswell (2004: 21) bereits bei den Humangeographen Yi-Fu Tuan und Edward Relph auf. 
Hinzu kommt ein dritter Mitspieler: landscape. Während in den oben zitierten Ausführungen von Johnstone (2010: 8) landscape gewissermassen eine Zwischenposition zwischen space und place einzunehmen scheint, ist dies bei Cresswell anders. Landscape bezieht sich im Vergleich zu den anderen zwei Begriffen am ehesten auf die materielle Komponente, doch schliesst auch dieses Konzept die individuelle Sicht der Menschen in Kontakt mit ihrer Umwelt ein. Landscape verbindet "a focus on the material topography of a portion of land (that which can be seen) with the notion of vision (the way it is seen)", schreibt Cresswell (2004: 10) und fährt fort: "Landscape is an intensely visual idea. In most definitions of landscape the viewer is outside of it. This is the primary way in which it differs from place. Places are very much things to be inside of'.2

Die deutschsprachige Diskussion setzt die Begriffe „Raum“ und „Ort“ ein, wobei z. B. nach der Soziologin Martina Löw „Ort“ näher beim Physisch-Materiellen steht. Löw (2012: 201) unterstreicht die Handlungskomponente bei der Konstitution von „Raum“. Dabei basieren „Raumkonstruktionen mittelbar oder unmittelbar auf Lokalisierung" und entstehen innerhalb eines „Prozeßes des Platzierens bzw. Platziert-Werdens“. Damit dieser Prozess überhaupt in Gang kommen kann, „muß es Orte geben, an denen platziert werden kann“ (Löw 2012: 198). Dennoch scheint auch bei Löw die Frage nach dem Verhältnis zwischen „Raum“ und „Ort“ nicht eindeutig geklärt zu sein.

Inwiefern die materiellen Gegebenheiten in die theoretischen Modellierungen miteinbezogen werden, ist nicht zuletzt eine Frage des Forschungsinteresses. In Anbetracht vieler linguistischer Fragestellungen (z. B. der Dialektologie oder der Variationslinguistik), für die die topographische Verortung des Datenmaterials durchaus von Relevanz ist, würde ich für eine Sicht plädieren, wonach die physisch-materielle Dimension vertreten sein sollte. ${ }^{3}$ Dabei darf sie weder mit der mentalen Dimension vermischt, noch gänzlich davon getrennt werden, sondern muss im Hinblick auf die dynamischen Beziehungen zwischen den beiden betrachtet werden. Um die Mehrdeutigkeit der Opposition „Ort“/,Raum“ zu umgehen, schlage ich vor, die materiell-topographischen Gegebenheiten als „Lokalitäten“ zu bezeichnen. Solche Lokalitäten sind z. B. die hier untersuchten Ortschaften Altdorf, Schwyz, Sarnen und Stans. Das Vorhandensein solcher Lokalitäten und die damit verbundenen Erfahrungen und Eindrücke spiegeln sich in ihren mentalen Repräsentationen wider. Doch auch Weltwissen, Vorlieben, Einstellungen etc., die nur indirekt mit der Lokalität zu tun haben, fliessen mit ein. So haben verschiedene Personen auch unterschiedliche Vorstellungen von einer Ortschaft wie beispielsweise Bad Säckingen oder Genf. Die mentalen Repräsentationen sind diejenige Komponente, die als Raum bezeichnet werden kann. Um auch hier Mehrdeutigkeit zu umgehen, wer-

\footnotetext{
2 Dass hier erneut die „Container“-Sicht auf Räume aufscheint, zeigt, wie sehr sie in unserem Denken verwurzelt ist: Bei der Übernahme der Perspektive eines Individuums schlüpft der Forscher automatisch in dessen Denkwelt, die eine Vorstellung des „im-Raum-seins“ vs. „ausserhalb eines Raumes-seins“ enthält. Inwiefern diese Sicht nicht ebenfalls eine gewisse Berechtigung hat und der Wahrnehmung des Menschen als körperhaftes Wesen entspricht (Stichwort embodiment), kann an dieser Stelle nicht weiter diskutiert werden.

3 Die nachfolgenden Ausführungen wurden im Ansatz in Petkova (2015) vorgestellt und werden an dieser Stelle wieder aufgegriffen.
} 
de ich von „mentalen Raummodellen“ sprechen. ${ }^{4}$ Das mentale Raummodell wiederum ist der Ausgangspunkt von raumbezogenem Handeln, das verschiedene Ausprägungen haben kann: Von der Denkmalpflege bis hin zum Bau von Autobahnen und futuristischen Gebäuden. Für die Linguistik von Interesse ist in erster Linie das ,raumbezogene sprachliche Handeln“. Dieses äussert sich z. B. als Einsatz bestimmter sprachlicher Strategien, die Ortsgebundenheit oder - im Gegensatz dazu - ein weltgewandtes Image signalisieren (Realisierung bestimmter lautlicher Variablen, Lexemwahl, Code-switching etc.). Die untenstehende Graphik illustriert diese Verhältnisse. ${ }^{5}$ Essentiell dabei ist die Wirkungsrichtung: Einzig zwischen dem mentalen Raummodell und dem raumbezogenen Handeln können Wechselwirkungen bestehen. Dagegen kann sich das mentale Modell einzig über das Handeln auf die Lokalität auswirken, während die Lokalität das Handeln einzig durch die Ausprägung oder Veränderung eines mentalen Modells beeinflussen kann.

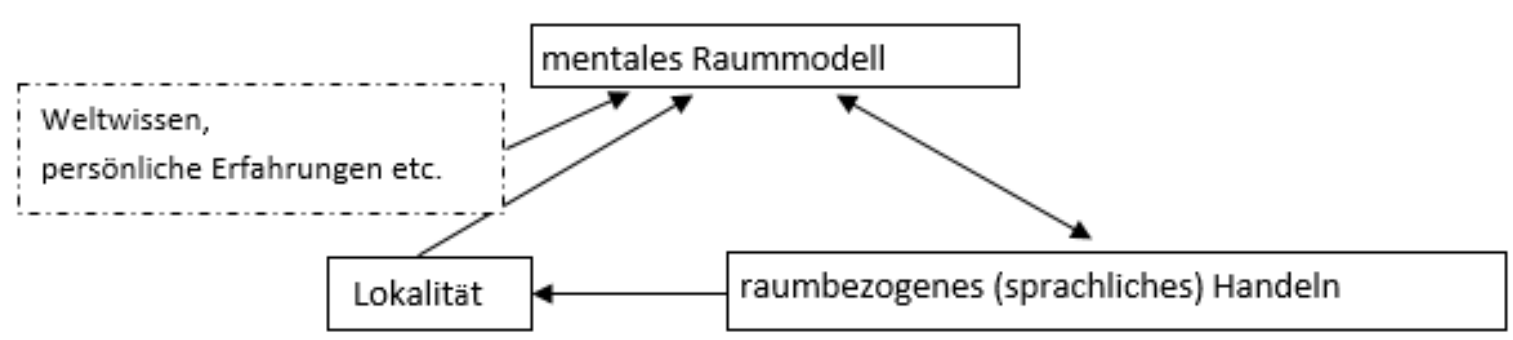

Grafik 1: Das Verhältnis zwischen Lokalität, mentalem Raummodell und raumbezogenem (sprachlichen) Handeln

Mentale Modelle sind dynamisch und werden ständig aktualisiert und gegebenenfalls verändert (ausführlicher hierzu Rickheit/Weiss/Eikmeyer 2010, Kap. 3.2.4). In einem engeren Sinne kann sich der Begriff place-making auf die Wechselwirkungen zwischen mentalem Raummodell und (sprachlichem) Handeln beziehen. Das Handeln kann sich aber auch auf die Lokalität auswirken, diese verändern, ergänzen oder beeinflussen. In der hier vertretenen Sicht, wonach die Verhältnisse zwischen Lokalität, mentalem Raummodell und raumbezogenem Handeln das Forschungsinteresse bestimmen sollten, wäre eine weite Auffassung von place-making denkbar als die Summe aller Prozesse, die sich zwischen allen dreien Komponenten abspielen. Die Unterscheidung zwischen space und place, wie insbesondere in Gieryn (2000) und Cresswell (2004) definiert, betrifft das Vorhandensein von mentalen Raummodellen mit unterschiedlichem Wert. Eine solche Unterscheidung ist insbesondere dann von Relevanz, wenn die gleiche Lokalität durch Aktualisierung und Modifizierung des mentalen Modells von einem neuen mentalen Modell repräsentiert wird. Dies geschieht dann, wenn sich für jemanden infolge eines Umzugs oder neuer privater oder geschäftlicher Beziehungen ein bedeutungsarmes space-Modell in ein emotional geladenes place-Modell verwandelt (dass das Verhältnis zwischen den beiden als kontinuumsartig zu betrachten ist, wurde bereits an-

\footnotetext{
${ }^{4}$ Rickheit/Weiss/Eikmeyer (2010: 39) definieren „,mentale Modelle“ als ,ganzheitliche, strukturerhaltende interne Repräsentationen externer Objekte, Sachverhalte oder Ereignisse“. Ein mentales Modell geht über die blosse Denotation hinaus, hat holistische Struktur und bezieht auch sprachunabhängiges Weltwissen mit ein.

5 Die hier vertretene Position steht in einer Linie mit Lefebvres (1980) Unterteilung in « espace perçu », « espace conçu » und « espace vécu » und Sojas (1996) "trialectics of spaciality" unterteilt in "firstplace", "secondplace" und "thirdplace". Auf Ähnlichkeiten und Unterschiede kann hier nicht im Detail eingegangen werden.
} 
gedeutet). Dieser Aspekt macht eine andere Dimension der Grösse „Raum“ sichtbar: ihre prozesshafte, zeitliche Komponente. „Raum“ ist ständigem Wandel unterzogen, die mentalen Raummodelle befinden sich als eigentliche Raum/Zeit-Einheiten in fortwährenden Aktualisierungsprozessen.

Die linguistic landscape setzt sich aus den sichtbaren, ins Physisch-Materielle eingegangenen Auswirkungen des raumbezogenen sprachlichen Handels zusammen. Vordergründig wird sie in der obigen Grafik also durch die Lokalität repräsentiert. Dahinter stehen jedoch ganz bestimmte, für den jeweiligen Kontext relevante mentale Raummodelle. Deren Aktualisierung bzw. Ergänzung führt zu weiteren Akten von raumbezogenem Handeln, was wiederum die Lokalität und damit auch die linguistic landscape beeinflusst.

\section{$3 \quad$ Linguistic landscapes (LL): Begriffsbestimmung}

Dass die physisch-materielle Dimension Eingang in die theoretische Modellierung des Verhältnisses zwischen Sprache und Raum finden sollte, wird besonders deutlich bei der Beschäftigung mit den linguistic landscapes. Diese neue Subdisziplin der Linguistik betrachtet "the use of language in its written form in the public sphere" (Gorter 2006: 2), um eine der vielen ähnlich ausgerichteten Definitionen in der aktuellen Diskussion zu zitieren (cf. u. a. auch Ben-Rafael et al. 2006: 7, Shohamy/Gorter 2009: 1, Auer 2010: 273). Ihren Ausgangspunkt hat die Beschäftigung mit linguistic landscapes (nachfolgend abgekürzt als LL) in den Beiträgen von Spolsky und Cooper (1991) und insbesondere von Landry und Bourhis (1997). Letztere haben auch den Terminus geprägt, der den Namen der Forschungsrichtung gibt.

Die vielzitierte erste Definition von Landry/Bourhis (1997: 25) bietet jedoch eher eine Aufzählung möglicher Ausprägungen, als eine genaue Bestimmung des Forschungsobjekts: "The language of public road signs, advertising billboards, street names, place names, commercial shop signs, and public signs on government buildings combines to form the LL of a given territory, region, or urban agglomeration”. Der Terminus wurde ebenfalls Gegenstand von Kritik, weil er in seinen beiden Bestandteilen vordergründig das Forschungsobjekt nur unzureichend erfasst. Zum einen war das Forschungsinteresse lange Zeit auf grossstädtische Umgebungen gerichtet. Dabei wurde sogar postuliert, dass ausschliesslich die Zentren der urbanen Grossstädte in den Blick genommen werden sollten (Ben-Rafael 2009: 41). Eine Reihe von Forschern (u. a. Gorter 2006: 2, Spolsky 2009: 25) weisen denn auch darauf hin, dass das Forschungsobjekt nicht als landscape, sondern vielmehr als cityscape zu erfassen wäre. Auf der anderen Seite führen Jaworski/Thurlow (2010: 19) mit Bezug auf den Humangeographen David Harvey aus: Die "invention and reinvention of places is an intensely complex social process, which includes but [...] does not privilege language and discourse". So sprechen Jaworski/Thurlow (2010) von "semiotic landscapes", Scollon und Wong Scollon (2003) beschreiben ihr Interessensgebiet als „Geosemiotik“, während Ziegler (2013) als Alternativterminus „Sehflächen“ vorschlägt. Der Begriff linguistic landscape hat sich dennoch etabliert, wobei eine Abkehr davon vielleicht auch nicht notwendig ist. Denn einerseits kann landscape, wie bereits deutlich wurde, verschiedene Lesarten evozieren. Eine weitere fügen Jaworski/Thurlow (2010: 5) hinzu, wenn sie landscape in einem weitesten Sinne als Metapher verstehen, als "text and spectacle”, und zugleich als ein „diskursives Terrain“, auf dem Identitätsempfinden, soziale Beziehungen und Machtverhältnisse ihre Spur hinterlassen. Gleichzei- 
tig hat die Linguistik in den letzten Jahrzehnten nebst der Sprache eine deutliche Öffnung hin zu weiteren Zeichensystemen vollzogen (Stichwort „Multikodalität“ bzw. „Multimodalität“). Der Miteinbezug von Bild, Schrift, materieller Trägerschaft etc. erhält ein immer grösseres Gewicht und kann mittlerweile als selbstverständlich gelten.

\section{Der Untersuchungsgegenstand}

Im Zentrum des Interesses der LL-Forschung standen lange Zeit Fragen nach dem Ausdruck von Mehrsprachigkeit und nach der Repräsentation verschiedener Sprachgruppen im öffentlichen Raum. Dabei wurden wie bereits erwähnt einerseits grossstädtische Umgebungen, andererseits meist Sprachen mit grossem strukturellen Abstand zueinander - häufig einschliesslich unterschiedlicher Schriftsysteme - in den Blick genommen (cf. exemplarisch die Arbeiten von Ben-Rafael et al. (2006) zu Hebräisch, Arabisch und Englisch in Jerusalem, von Huebner (2006) zu Thai, Chinesisch und Englisch in Bangkok oder von Backhaus (2007) zu Japanisch, Englisch, Chinesisch und Koreanisch in Tokio). ${ }^{6}$ Die hier vorgestellte Studie geht einen anderen Weg. Einerseits interessiert sie sich nicht für die Machtverhältnisse zwischen bestimmten sozialen Gruppen, sondern für den Ausdruck von Zugehörigkeitsempfinden zu bestimmten Räumen. Von Bedeutung ist hier vor allem die Frage danach, welche Ebenen der (arealen) Zugehörigkeit kategoriell angesprochen werden: Lokale Zugehörigkeit (Gemeinde), kleinregionale (Kanton), grossregionale (Region bzw. ganze Deutschschweiz) oder sprachregionale (Dialektgebiet), nationale (Schweiz) oder internationale Zugehörigkeit. Hierbei stellt die Code-Wahl ein Ausdrucksmittel unter anderen dar. Der Blick richtet sich zudem auf vier relativ kleine Gemeinden: Altdorf (Hauptort von Uri), Schwyz (Hauptort von Schwyz), Sarnen (Hauptort von Obwalden) und Stans (Hauptort von Nidwalden). ${ }^{7}$ Die Gemeinden beherbergen die Behörden und Institutionen des jeweiligen Kantons (kantonale Verwaltung, Polizei etc.). Bezüglich Fläche und Bevölkerung hingegen sind sie klein, es handelt sich also um Ortschaften, deren Bewohner sie womöglich selbst nicht einmal als Städte bezeichnen würden (hierzu mehr weiter unten).

Die vier Kantone Uri, Schwyz, Nid- und Obwalden (die beiden letzten wurden früher auch als Unterwalden zusammengefasst) gelten als dasjenige Gebiet, auf dem das erste eidgenössische Bündnis entstanden ist. Die Bezeichnung „Urschweiz“ nimmt Bezug auf diese mythische Entstehungsgeschichte der Schweiz und dient zugleich als Toponym, mit dem die Region benannt wird. In dieser oder in einer grösseren Ausdehnung kann das Gebiet aber auch mit einem weiteren Toponym, „Innerschweiz“, bezeichnet werden. Ein mehrteiliges Assoziationsexperiment zu den mentalen Repräsentationen der beiden Toponyme „Urschweiz“ und „Innerschweiz" ergab, dass dahinter mentale Raummodelle stehen, die sich teilweise decken und teilweise unterscheiden, die sich aber grösstenteils auf die gleichen Lokalitäten beziehen (für eine detaillierte Darstellung von Methode und Ergebnissen cf. Petkova 2015). Das Assoziationsexperiment war auf die Aussenperspektive ausgerichtet: Die Teilnehmenden waren un-

\footnotetext{
6 Diese Ausrichtung der Disziplin zusammenfassend postuliert denn auch Spolsky (2009: 25): “One of the main topics of interest is the choice of language in public signs in bilingual or multilingual urban space."

7 Die hier vorgestellte Studie entstand im Rahmen des vom Schweizerischen Nationalfonds unterstützten Forschungsprojekts Ländere ${ }^{n}$ - die Urschweiz als Sprach(wissens)raum an der Universität Freiburg (CH). Die Wahl der untersuchten Region steht in Zusammenhang mit der Projektthematik.
}

ISSN 1615-3014 
terschiedlicher schweizerischer Herkunft. Die hier vorgestellte Studie vertritt eine andere Sichtweise. Ausgehend von den Effekten des raumbezogenen sprachlichen Handelns, die sich in materialisierter Form in der Lokalität vorfinden, erkundet sie, wie sich die Bewohner/innen der betreffenden Gemeinden selbst positionieren.

\section{$5 \quad$ Methodik}

Während in der LL-Forschung die Materialgewinnung dank den Fortschritten der Digitalfotographie keine besonderen Schwierigkeiten bereitet, steht die Methodik der Disziplin immer noch vor grösseren Herausforderungen. Die Diskussion über Möglichkeiten und Grenzen der methodischen Verfahren ist entsprechend im Gange. Androutsopoulos (2014: 83) nennt drei Grundsatzentscheidungen, die bei einer Untersuchung getroffen werden müssen: die Wahl des Untersuchungsareals, die Wahl der untersuchten Elemente und die Wahl der Kategorien, nach denen sie klassifiziert werden. Alle drei Entscheidungen bergen Probleme. So unterscheidet sich z. B. die LL bereits in der gleichen Stadt wesentlich von Stadtteil zu Stadtteil, sodass je nach Arealwahl die gleiche Forschungsfrage unterschiedliche Antworten erhalten kann. Die untersuchten Elemente wiederum sind manchmal so angeordnet, dass die Entscheidung darüber, wo ein Zeichen aufhört und wo das nächste beginnt, nicht eindeutig getroffen werden kann. "One needs to make ad hoc decisions about boundaries, raising problems for the reliability of counts", schreibt hierzu Spolsky (2009: 32, kursiv im Orig.) Die berücksichtigten Kategorien können von fest montierten Schildern bis hin zu Aufklebern und Graffiti reichen. Welche und wie viele Kategorien in die Untersuchung miteinbezogen werden, wirkt sich einerseits auf die Arealgrösse aus, die abgedeckt werden kann. Eine Rolle spielt aber auch der Umstand, dass viele Elemente flüchtig sind und häufige Wechsel stattfinden. So gleicht jede Studie, die die Diversität der LL möglichst umfassend abbilden möchte, immer einem punktuellen Schnappschuss. Die Berücksichtigung nur dauerhafter Zeichen wiederum schränkt die Aussagekraft der Untersuchung für viele Fragestellungen ein. ${ }^{8}$

Die hier vorgestellte Studie wählt eine Vorgehensweise, die keine Ansprüche auf die Abbildung der gesamten LL (auch nur eines Stadtteils oder einer Strasse) erheben kann. Die Materialsammlung richtet sich nach der gewählten Fragestellung, für die nur bestimmte Zeichen von Relevanz sind. So wurden in erster Linie Toponyme berücksichtigt, aber auch die in $\mathrm{Zu}-$ sammenhang damit stehenden Wappen, Flaggen u. Ä. Weiter war das Vorkommen von dialektalen Anschriften von Interesse. Ergänzend dazu kamen Bilder, weitere Sprachen u. a. Insofern als die LL einer Ortschaft ständig im Wandel begriffen ist, kann auch der Zeitpunkt der Datenerhebung eine Rolle spielen. Dies ist nicht immer nachteilig: Bestimmte Anlässe bewirken eine Anreicherung der LL, die der Materialgewinnung zugutekommen kann. Im Zusammenhang mit dem Schweizer Nationalfeiertag am 1. August werden die Strassen geschmückt und die Schaufenster auf den Anlass hin dekoriert. Es werden auch verschiedene Produkte zum Verkauf angeboten und ausgestellt, die auf den Anlass und auf die Lokalität Bezug nehmen. Daher eignen sich die Tage unmittelbar vor dem Nationalfeiertag gut für eine Materialsammlung, die sich auf die hier interessierende Fragestellung hin ausrichtet, insbesondere was

\footnotetext{
8 Für weitere methodische Überlegungen cf. die Beiträge in Shohamy/Gorter (2009), insbesondere in Part I, Theoretical Perspectives und Part II Methodological Issues.
} 
die Zusammenstellung von heraldischen Zeichen betrifft. So besuchte die Autorin am 31. Juli 2013 Altdorf und am 30.-31. Juli 2014 Schwyz, Sarnen und Stans. ${ }^{9}$ Der Rundgang hatte eine Dauer von jeweils 4-6 Stunden pro Gemeinde. Nach der Ankunft am Bahnhof wurde jeweils das Gemeindezentrum als Kern und Treffpunkt der Gemeinde besucht. Danach wurden im Tourismusbüro Informationen darüber eingeholt, welche Sehenswürdigkeiten den Gästen empfohlen werden. ${ }^{10}$ Altdorf und Schwyz verfügen zudem über Touristenrundgänge, wobei an jedem entsprechenden Punkt auf der Route Schrifttafeln angebracht sind. ${ }^{11}$ Der Miteinbezug der touristischen Empfehlungen stützte sich auf die Annahme, dass sich die Gemeinde an diesen Orten selbst präsentiert und ein gewissermassen kontrolliertes Image aufbaut, das in der Untersuchung berücksichtigt werden muss. Der weitere Gang durch die Ortschaften hatte zum Ziel, möglichst viel Fläche vom Zentrum aus zur Peripherie hin abzudecken und Zeichen mit unterschiedlicher Urheberschaft miteinzubeziehen. Die Materialsammlung beschränkte sich auf öffentliche Orte, die sich , unter freiem Himmel‘ befinden (also ohne die Innenansicht von Museen, Verwaltungsgebäuden etc.).

\section{Ergebnisse}

Zugehörigkeit kann in der LL auf zweierlei Wege zum Ausdruck kommen. Einerseits kann inhaltlich darauf Bezug genommen werden, z. B. indem eine Bezugsgrösse explizit genannt wird. Andererseits kann z. B. die Sprachwahl Hinweise auf das Zugehörigkeitsempfinden liefern. In diesem Sinne kann die Inhaltsseite oder die Ausdrucksseite der Zeichen Zugehörigkeit markieren (für Beispiele aus einem anderen Deutschschweizer Kontext s. Christen et al. 2010, Kap. 4).

\subsection{Ausdrucksseitige Zugehörigkeit: Dialektale Anschriften}

Während Auer (2009: 1) davon ausgeht, dass "dialects do not usually play a part in the formation of the linguistic landscape", kann angenommen werden, dass der Dialekt in der LL der Deutschschweiz aufgrund der besonderen Stellung der Varietät vergleichsweise häufiger anzutreffen ist (zum Verhältnis zwischen Dialekt und Standard in der Deutschschweiz s. Am-

\footnotetext{
${ }^{9}$ Die Vorgehensweise, die nicht nur eine blosse Materialsammlung, sondern auch die beobachtende Auseinandersetzung mit den Lokalitäten einschloss, bedingte es, dass an allen Untersuchungsorten die gleiche Person zum Einsatz kommt. Da zwischen den untersuchten Gemeinden z. T. längere Reisewege liegen, liess es sich nicht vermeiden, die Datenerhebung auf zwei Jahre zu verteilen. Der 1. August selbst eignet sich für die Datenerhebung nicht, da einerseits aufgrund der Feierlichkeiten viele Strassen gesperrt sind, andererseits die Geschäfte schliessen und weniger Auslagen in den Schaufenstern stehen.

${ }^{10}$ Die einzige Gemeinde, die über kein Tourismusbüro verfügt, ist Stans. Hier erfüllt der Bahnhofsschalter diese Funktion.

11 Aufschlussreich für das Selbstverständnis der untersuchten Gemeinden ist die Bezeichnung des Rundgangs in Schwyz: „Dorfrundgang“. Dies belegt exemplarisch, dass die Gemeinden, von denen Schwyz die bevölkerungsreichste ist, kaum als Städte wahrgenommen werden möchten und weit von jeder Urbanität stehen. Hierzu ist auf der Homepage von Schwyz zu lesen: „Gemäss eidgenössischer Statistik kommt ihm [dem Hauptort Schwyz, M.P.] gemessen an der Bevölkerung der Rang einer ,Stadt` zu. Gleichwohl hat Schwyz den historischen Charakter eines ,Fleckens' bewahrt, wenn auch seit einiger Zeit, nach jahrzehntelanger Stagnation, eine dynamische Entwicklung stattfand“". (www.gemeindeschwyz.ch/xml_1/internet/de/application/d2/d13/f14.cfm. [17.3.2016]).
}

ISSN 1615-3014 
mon 1995). ${ }^{12}$ Das Datenmaterial der hier vorgestellten Untersuchung bestätigt diese Annahme (für einige Beispiele s. Abb. 1 im Anhang). In der LL der untersuchten Gemeinden stehen dialektale Anschriften als Namen z.B. von Geschäften („Bluämä-eggä“, „Chäs Ladä“, „Gschänk Eggä“, „Schwyzer Milchhuus“, „Muisigdeesli“, „Huis-Ladä“ etc.), als Produktbezeichnungen („Chrüter-Häxli“ - Käse, „Schwyzer Chäs“ - Käse, „Ürner Chritermischig“ Tee, „Bürä-Biräwegge“ - Gebäck, „Senne-Chäppli“ - Gebäck, „Haussalat ,usem Schisseli“““ etc.), auf Veranstaltungsplakaten („14. Strassäfäscht“, „Obwaldner Chinderopenair“ etc.), in Slogans („Guets vo hie“ - Menüvitrine eines Restaurants, „Mmh... so guet!“ - Bäckereitafel, „Immer guet“ - Metzgereitafel, „Brot und Siässes wiä friäner“ - Bäckereiplakat, „Rageetä Wätter“" - Plakat im Schaufenster eines Optikergeschäfts etc.).

\section{Dialektale vs. Nicht-dialektale Elemente}

Die Anordnung der dialektalen Elemente gegenüber nicht-dialektalen Elementen (Standardsprache oder auch anderen Sprachen) lässt verschiedene Muster erkennen. Dialektale Namen stehen häufig für sich alleine („Teelädeli“, „Natur-Lädeli“, „Chinderhuus Sunnestrahl“, „Bluämä-eggä“, „Gschänk Eggä“, „Schwyzer Milchhuus“, „Muisigdeesli“, „HuisLadä“). Zuweilen bestehen Namen aus einem dialektalen und einem standardsprachlichen Teil („Chinderhuis Nidwalden“,"13 „Haus Plätzli“, „Mühligasse“, "Gastwirtschaft zum Briggli“"). Es kann vorkommen, dass dialektale Elemente auf der gleichen Granularitätsebene ${ }^{14}$ eine präzisierende standardsprachliche Ergänzung erhalten („Chäslager Stans Kulturhaus“, 15 „Reisebüro Feriezyt", „Guets vo hie, Gastro Nidwalden“"). Erklärendes findet sich zudem als standardsprachlicher Zusatztext, der auf einer niedrigeren Granularitätsebene steht, kleiner, in einer anderen Schrift geschrieben und/oder untenstehend platziert (,Immer guet Bio Lammfleisch vom Bogigenhof“ - Metzgereitafel, „Chäs Hüttä. Div. Käse, Weine, Spirituosen, Geschenkideen, Milchprodukte“ - auf dem Schaufenster des Ladens, „Chrüter-Häxli, fein zu Kartoffeln oder aufs Brot" - Tafel vor der gleichen Käserei, „Schwyzer Milchhuus. Ihr Schweizer Käsehaus“ - auf einem Plakat im Schaufenster, „Raucherbeizli. Auch Nichtraucher herzlich willkommen!“ - auf einer Tafel an der Aussenwand eines Restaurants, „Ürner Chritermischig, Ziehzeit 8-10 Minuten“" - Menükarte in der Vitrine eines Cafés). Aber auch das umgekehrte Muster lässt sich beobachten, z. B. auf der vor einem Restaurant aufgehängten Menükarte: „Rumpsteak mit Kräuterbutter, Pommes frites und Gemüse (vom feinsten vo de Metzgeri Stutzer)“ oder „Gemischter Tellspiess mit Pommes frites und Gemüse (e chli

12 Auer (2009) nimmt darauf in den Blick ein „seltenes Beispiel“ aus dem deutschen Kontext und belegt, dass der Einsatz des Dialekts eine symbolische Funktion hat und dazu dient, den Schwarzwald mit einem besonderen Image zu inszenieren. In diesem Sinne nimmt auch hier das raumbezogene sprachliche Handeln Bezug auf ein bestimmtes mentales Raummodell: Die Sprachwahl stellt die betreffende Lokalität in ein romantischrückwärtsgewandtes Licht.

13 Die standardsprachlichen Elemente in den Beispielen werden jeweils unterstrichen.

14 Auer (2010: 208f) definiert als Zeichen mit hoher Granularität solche, „die aus großer Entfernung gelesen werden können und die übergeordneten Handlungszielen [...] zugeordnet sind. [...] Geringe Granularität schränkt entweder die Gruppe der intendierten Rezipienten ein und selegiert dazu spezifischere soziale Rollen der Adressaten, oder sie dient subsidiären Handlungen innerhalb eines größeren Handlungsschemas“.

15 „Kulturhaus“ folgt unmittelbar nach „Chäslager Stans“ in der gleichen Schriftgrösse, ist im Gegensatz zum vorangehenden Teil aber nicht fett markiert und gehört nicht zum Namen, sondern dient als präzisierende $\mathrm{Zu}$ satzbezeichnung. 
Chipolata, Rind, Schwein und Speck $)^{“ .}{ }^{16}$ Auffallend in den beiden letzten Beispielen ist, dass die beschreibenden Zusätze nicht ganz in Dialekt gehalten sind, sondern auch auf dieser Ebene Dialekt und Standard nebeneinander auftreten. Ähnliches findet sich auf einer Bäckereitafel, wobei hier der Code-Wechsel sogar inmitten des Adjektivs stattfindet: „Mmh...so guet! gluschtige Mandelgipfel und Nussgipfel “. Im nächsten Beispiel aus einer Menükarte ist der Zusatz wiederum gänzlich in Dialekt, er beschreibt jedoch nicht das Gericht näher, sondern ahmt einen persönlichen Kommentar nach, dient in diesem Sinne zur Inszenierung einer zweiten Stimme und intendierter Mündlichkeit: „Hackbraten mit Röstikroketten und Gemüse. Da hätt de Grossdädi ai Freyd“. Interessante Kombinationen ergeben sich dort, wo weitere Sprachen hinzukommen. So steht z. B. auf der Tafel vor einem Café in Standard, Dialekt und Niederländisch: „Ab 11 Uhr warme Küche. Ä guätä! Eet smakelijk!“‘. Im Schaufenster einer Metzgerei mit dazugehörendem Restaurant hängen vorgefertigte Kreidetafeln mit den Namen der Wochentage auf Französisch. Die Flächen darunter sind von Hand ausgefüllt mit den unterschiedlichen Tagesangeboten teils in Standard, teils in Dialekt, z. B. „Lundi. Feini Würscht“ oder „Mercredi. gHacket \& Hörnli“, aber „Samedi. Offen bis 15.00“. Das Angebot einer Käserei wird auf verschiedenen Tafeln vor dem Eingang angepriesen, dicht neben der Tafel zum „Chrüter-Häxli“ (s. o.) steht eine weitere Tafel, auf der „Grill Cheese, Antipasti und Barbecue" 17 aufgezählt werden. Das Verhältnis zwischen Dialekt und Standard bzw. anderen Sprachen in mehrsprachigen Anschriften scheint also im Allgemeinen keinem festen Muster zu folgen, sondern wird variabel gehandhabt.

\section{Wahl der dialektalen Merkmale}

Bei der Wahl der dialektalen Elemente und bei ihrer Umsetzung in die Schriftlichkeit fallen einige Strategien auf, die immer wieder zum Einsatz kommen. Diese betreffen verschiedene sprachliche Ebenen - die Lautung, die Morphologie oder die Orthographie.

\section{- $k$-Verschiebung}

Die $k$-Verschiebung im Anlaut gehört zu den dialektalen Merkmalen, die die hoch- und höchstalemannischen Dialekte auszeichnen, wie sie in der Deutschschweiz gesprochen werden. Hier sorgt zudem bei der Verschriftlichung die Position am Anfang des Wortes für gute Sichtbarkeit. Das Merkmal findet sich in sehr vielen dialektalen Anschriften: „Chinderhuus“ bzw. „Chinderhuis“, „Chäs Hüttä“, „Ürner Chritermischig“, „Chäslager Stans“, „roter Chlais“, „Chinderopenair“, „Senne-Chäppli““ etc.

- $\quad$ Mhd. , , $\overline{\mathrm{u}}^{\prime \prime}$ als $u i$ (Ob- und Nidwalden) bzw. als $\ddot{u}$ (Uri)

Die Realisierung von mhd. „, $\overline{\mathrm{u}}^{\prime}$ als $u i$ (Ob- und Nidwalden) bzw. als $\ddot{u}$ (Uri) gehört zu den Besonderheiten der Dialekte in der untersuchten Region (SDS, Bd. I, Karte 142). Die Merkmale finden sich wiederholt in den Anschriften: „Huisladä“, „Muisigdeesli“, „Obwaldner Huismuisig“, „Chinderhuis“" etc. bzw. „Ürner Chritermischig“.

16 Hier ist der Teil „e chli“ eindeutig dialektal, der Teil „Chipolata, Rind“ lässt sich weder eindeutig dem Dialekt, noch dem Standard zuordnen. Die Lexeme Schwein und Speck dürften mit einiger Sicherheit standardsprachlich intendiert sein.

17 Die Lexeme Antipasti und Barbecue dürften als etablierte Entlehnungen im Deutschen gelten, beim Lexem Cheese ist dies nicht der Fall. 
- Vokalentrundung

Die Vokalentrundung gehört ebenfalls zu den regionalen Besonderheiten (SDS, Bd. I, Karte 102) und findet sich in mehreren Anschriften („Gastwirtschaft zum Briggli“, „Haussalat ,usem Schisseli““, „Muisigdeesli““, „Ürner Chritermischig“). ${ }^{18}$

- Diminutivsuffix -li

Das Diminutivsuffix - $l i$ ist charakteristisch für die Deutschschweizer Dialekte. Durch die Stellung am Wortende stellt es zudem ein weiteres gut sichtbares Merkmal dar und eignet sich somit gut, um die Markiertheit der dialektalen Anschriften zu unterstreichen. Im Korpus finden sich viele Beispiele: „Restaurant Höfli““, „Natur-Lädeli“, „Haus Plätzli““, „Raucherbeizli““, „Schwyzerstubli“, „Uristierli“" etc.

- Orthographische Verfremdung bei der Dialektverschriftlichung

Die orthographische Verfremdung gehört zu den Strategien, die bei der Verschriftlichung des Dialekts dazu eingesetzt werden, um eine maximale Abweichung vom standardsprachlichen Schriftbild, grösstmögliche Auffälligkeit und dadurch Signalwirkung zu erzielen (zu den Strategien der Dialektverschriftlichung Lötscher 1989, Christen 2004). Besonders hervorstechend und daher für solche Zwecke gut geeignet sind Umlautschreibungen des Schwa-Lauts und Buchstabenverdoppelungen. Diese Verschriftlichungsstrategie prägt viele Anschriften: „Bluämä-eggä“, „Bürä-Biräwegge“, „Grüüveli Tüüveli“, „Brot und Siässes wiä friäner“, „Ä guätä!‘ etc.

\section{Der Einsatz des Dialekts in seiner Bedeutung}

In der Deutschschweiz dient die Wahl des Dialekts in Kontexten, in denen die Standardsprache als die unmarkierte Varietät gilt, häufig dazu, Zugehörigkeit und Identifizierung mit einem bestimmten dialektalen Gebiet oder mit dem Landesteil als Ganzem zu signalisieren. Von besonderer Bedeutung in unserem Kontext ist der Umstand, dass die dialektalen Merkmale in den Anschriften zu denjenigen gehören, die aus der Laiensicht als besonders salient gelten dürften. Dabei weisen sie in zwei unterschiedliche Richtungen. Zum einen handelt es sich um Merkmale, die mit den Deutschschweizer Dialekten im Allgemeinen in Verbindung stehen. Ein solches Merkmal ist die anlautende $k$-Verschiebung. Wahrnehmungsdialektologische Untersuchungen zeigen, dass dieses Merkmal nicht zuletzt auch aus der Aussensicht ,als das herausragende Erkennungszeichen des Schweizerdeutschen betrachtet werden kann“ (Stoeckle 2015: 461, hierzu auch Schifferle 2012: 183). Es ist ebenfalls davon auszugehen, dass ein Merkmal wie das Diminutivsuffix -li einen hohen Bekanntheitsgrad aufweist und dadurch als Unterscheidungsmerkmal innerhalb des deutschsprachigen Raums und als Verweis auf die Deutschschweiz dienen kann. Auch die orthographische Verfremdung zielt nicht primär auf die areale Zuordnung bestimmter Merkmale, sondern auf die Sichtbarmachung des Dialekts im Allgemeinen.

Auf der anderen Seite stehen Merkmale, die als salient für die untersuchte Region empfunden werden. Wie die wahrnehmungsdialektologisch ausgerichtete Befragung des Forschungspro-

18 Für die beiden letztgenannten Merkmale gilt jedoch, dass sie nicht ausschliesslich in den genannten Regionen anzutreffen sind.

ISSN 1615-3014 
jekts „Ländere ${ }^{\text {n“ }}$ belegt, werden für Ob- und Nidwalden die Realisierung des mhd. „, „“ als $u i$, die Vokalentrundung oder das Diminutivsuffix (-ili vs. -eli) besonders häufig als typische Merkmale genannt (für eine ausführliche Darstellung von Untersuchungsanlage und einigen Resultaten cf. Schiesser in Vorb.).

Viele der angeführten Beispiele zeigen zudem eine weitere Strategie, die auf die Lexemwahl ausgerichtet ist. In den dialektalen Anschriften der untersuchten LL finden sich besonders häufig einige wenige Lexeme des Grundwortschatzes wie „Haus“, „Kind“, „Käse“, „Laden“ oder „Musik“. In vielen Fällen charakterisieren diese die Bestimmung der Gebäude, an denen die Anschriften angebracht sind. Da es sich dabei meist um Geschäfte oder Kindertagesstätten handelt, ist deren Funktion aber bereits anhand von Schaufenstern, Wanddekorationen u. Ä. ersichtlich. Dadurch dürften auch Dialektunkundige kaum Schwierigkeiten damit haben, den Inhalt der Anschriften zu verstehen. Der Dialekt wird also nicht aus einer Notwendigkeit heraus eingesetzt, z. B. um lexikalische Lücken in der Standardsprache zu füllen, sondern dort, wo die Sprache nebst anderen Codes sozusagen redundant ist. Die benutzten Lexeme sind ausserdem so frequent und vertraut, dass sie auch in einer abgewandelten Form schnell entschlüsselt werden können. So rückt die Sprachform in den Vordergrund: Die eigentliche Botschaft wird von der Sprachwahl transportiert. Um diese Botschaft zu interpretieren, lassen sich die theoretischen Konzepte des "enregisterment” (Agha 2003) oder der „Indexikalität" (Johnstone et al. 2006 in Anlehnung an Michael Silverstein) heranziehen. Agha (2003: 231) beschreibt das "enregisterment" als "processes through which a linguistic repertoire becomes differentiable within a language as a socially recognized register of forms". Dabei transportieren bestimmte Merkmale eine soziale Bedeutung. Diese kann unterschiedliche Abstraktionsstufen oder "orders of indexicality" erreichen (cf. Johnstone et al. 2006). Zunächst steht die "first-order indexicality" als potentielle Indexikalität, die erst zur Geltung kommt, wenn gewisse sprachliche Merkmale wahrgenommen werden und ihre Eignung als Träger von sozialer Bedeutung erkannt wird. Dies geschieht auf der nächsten Stufe, derjenigen der "secondorder indexicality". "Second-order indexicality occurs when people begin to use first-order correlations to do social work, either interpretive or performative" (Johnstone et al. 2006: 83). Die letzte Stufe, die "third-order indexicality", wird nur noch von wenigen der infrage kommenden Merkmale erreicht. Diese gewinnen jedoch hohen Wiedererkennungswert, werden auch metasprachlich thematisiert und konstituieren einen lebhaften, breit rezipierten Diskurs, worin sie nicht zuletzt lokale Identität symbolisieren. "The meanings of these forms are increasingly linked to place", schreiben Johnstone et al. (2006: 84). Die oben aufgelisteten dialektalen Merkmale, die sich in der LL der untersuchten Gemeinden finden, können als Merkmale mit "third-order indexicality" gelten. In ihrer materialisierten Form kennzeichnen sie die Lokalitäten als zu bestimmten Räumen zugehörig. Einer dieser Räume ist die Deutschschweiz, ein anderer die umliegende Kleinregion, die von spezifischen areal-sprachlichen Merkmalen evoziert wird.

\subsection{Inhaltsseitige Zugehörigkeit: Toponyme und weitere Zeichen}

\section{Toponyme}

Der Rundgang durch die untersuchten Gemeinden zeigte, dass in ausserordentlich vielen Anschriften Toponyme enthalten sind: sprachliche Einheiten, die den Bezug zu einer geographi- 
schen Grösse anzeigen. Diese können als Zusatz zu einem Geschäftsnamen daherkommen (z. B. „Hörmittelzentrale Stans“, „Hotel Engel Stans“), im Namen selbst enthalten sein (z. B. „Obwalden Tourismus“, „Sarner Fachgeschäfte“), einen Slogan bilden („Urner Power“ der Elektrizitätswerke Uri) oder in offiziellen Anschriften die institutionelle Urheberschaft angeben („Kantonspolizei Schwyz"). Die Bezugsgrösse solcher topographischer Verweise kann unterschiedlich sein: Von Gemeinde über Kanton, Region, Land oder auch auf geographisch weiter entfernte Orte referierend (z. B. "Dublin, the Irish Pub" in Altdorf). Das Vorhandensein und die Wahl dieser Grössen sind keineswegs selbstverständlich. Dies zeigt sich nicht nur in bottom-up-Zeichen (z. B. an Geschäften, Restaurants etc.), sondern auch in top-downZeichen (von institutioneller Seite angebrachten Tafeln und Anschriften). ${ }^{19}$ So führt die „Bäckerei-Conditorei-Café Frei Stans“ den Gemeindenamen im Logo, während der „Lehn-Beck“ in Altdorf das Wappentier des Kantons Uri, den Stier, in sein Logo integriert. Der Friseursalon „Coiffure Lara“ in Stans kommt ohne jeden topographischen Zusatz aus, während der Friseursalon „Coiffure Maya Henseler Schwyz“ den Gemeindenamen einmal auf dem vor dem Geschäft hängenden Schild und noch zweimal im Schaufenster trägt (für alle Beispiele s. Abb. 2). Ähnliches lässt sich auch bei den top-down-Zeichen beobachten: Während die Kantonale Verwaltung in Schwyz auf der davorstehenden Tafel mit „Kanton Schwyz“ angeschrieben ist, reicht bei der Kantonalen Verwaltung in Obwalden das Kantonswappen als Verweis aus. Hierbei muss daran erinnert werden, dass es sich bei den untersuchten Zeichen um solche handelt, die an einem bestimmten topographischen Punkt angebracht sind. Dass sich ein bestimmtes Geschäft in dem genannten Ort befindet, braucht man nicht erst aus der Anschrift zu erfahren: Um die Anschrift überhaupt lesen zu können, muss man selbst vor Ort sein. Dass die Sprache in der LL nicht nur eine informative, sondern auch eine symbolische Funktion hat, wird in der Literatur seit Landry/Bourhis (1997) immer wieder betont. Das Vorhandensein der beiden Funktionen ist in Beispielen wie den oberen besonders deutlich. Das Führen eines Toponyms im Logo oder die Adressenangabe auf der Tafel beim Eingang tragen in diesem Sinne weniger eine informative, sondern vor allem eine symbolische Bedeutung. ${ }^{20}$ Die toponymischen Angaben demonstrieren areale Zugehörigkeit. Wichtig ist dabei, dass es sich um eine Zugehörigkeit handelt, die anders ausgerichtet ist als z. B. beim Namen des Geschäftsbesitzers auf dem Schaufenster. Die Botschaft im letzten Fall ist: „Dieses Geschäft gehört Herrn oder Frau X“. Die Toponyme in den Anschriften hingegen bringen ein Zugehörigkeitsempfinden zum Ausdruck. Sie sagen: „Herr oder Frau X sehen sich als Geschäftsbesitzer zugehörig zum Raum Y“. Ob der Gemeinde-, der Landesname oder eine andere Grösse genannt wird, entscheidet das mentale Raummodell, das im Vordergrund steht. Gleichzeitig darf nicht ausser Acht gelassen werden, dass es sich in vielen Fällen um kommerzielle Zeichen handelt, die zum Ziel haben, eine bestimmte Kundschaft anzusprechen. In diesem Sinne könnte hier zugleich die Annahme der Geschäftsbesitzer über die Vorlieben der vorbeigehenden Kunden, eingeschlossen ihr vermutetes Zugehörigkeitsempfinden, im Spiel sein.

19 Zum Konzept der top-down- und bottom-up-Zeichen cf. Ben-Rafael et al. (2006), auf die berechtigte Kritik an diese Kategorien wird an dieser Stelle nicht eingegangen.

20 Im Übrigen gehören natürlich Firmenname, Adresse und Logo häufig zur corporate identity einer Firma und prägen ihren Auftritt in unterschiedlichen Kontexten, vom Briefpapier bis hin zur Hausanschrift.

ISSN 1615-3014 
Wie verteilen sich nun die geographischen Grössen im Datenkorpus? Um die Verhältnisse überblicken zu können, wurden zunächst alle Zeichen, auf denen Toponyme zu finden sind, sortiert und anschliessend nach Anteilen ausgewertet. Es wurden folgende Kategorien gebildet: „Gemeinde“ (bezieht sich auf die jeweilige Gemeinde, aus der die Daten stammen), „Kanton“ (bezieht sich auf den Kanton, dessen Hauptort die jeweilige Gemeinde ist), „Region“ (bezieht sich auf die übliche Zusammenführung von Uri, Schwyz, Obwalden und Nidwalden ev. zusammen mit weiteren Kantonen zu einer Region wie Urschweiz, Innerschweiz oder Zentralschweiz), „Land“ (bezieht sich auf die Schweiz unabhängig davon, in welcher Sprache die Bezeichnung ist), ,regionale Orientierung“ (bezieht sich auf Angaben zu weiteren Ortschaften in regionaler Nähe, häufig sind dies eine oder mehrere der anderen untersuchten Gemeinden), ,nicht-CH“ (nicht-schweizerische Bezüge). ${ }^{21}$ Gezählt wurden jeweils die einzelnen Zeichen. ${ }^{22}$ Mehrfache Nennungen des gleichen Toponyms im gleichen Zeichen wurden nicht berücksichtigt, das Zeichen wurde nur einmal gezählt. Nennungen unterschiedlicher Toponyme im gleichen Zeichen wurden berücksichtigt - das Zeichen wurde in diesem Sinne doppelt gezählt (z. B. im Falle von „Altdorf, Uri“ einmal als Angabe zu „Gemeinde“ und einmal als Angabe zu „Kanton“). Da im Korpus Zeichen von unterschiedlicher Granularität vorhanden sind und keine Granularitätsstufe wesentlich überwiegt, erfolgte die Zählung unabhängig von der Granularität der Zeichen. Wegweiser wurden nicht berücksichtigt, denn ihr Zweck besteht darin, die Richtung zu einer Ortschaft zu zeigen, wobei diese unweigerlich genannt werden muss. Eine grössere Schwierigkeit bei der Auswertung ergab sich daraus, dass im Kanton Schwyz der Kantonsname und der Name des Hauptortes identisch sind. Wenngleich einige Zeichen eine Zuordnung ermöglichen (wie z. B. in Falle der „Kantonalen Verwaltung“ oder wenn explizit „Gemeinde Schwyz“ angegeben ist), ist die Anzahl nicht eindeutiger Nennungen zu gross. Die Vermutung, dass sich viele von ihnen auf die Gemeinde beziehen, lässt sich anhand der Daten nicht erhärten. Daher wurde die Nennung „Schwyz“ als ambig, auf „Gemeinde“ oder „Kanton“ hinweisend, in einer Kategorie zusammengefasst. Für Altdorf, Stans und Sarnen ist hingegen eine differenzierte Auswertung möglich. Die untenstehende Grafik zeigt die Resultate der Auswertung.

\footnotetext{
21 Eine Kategorie „über die Region hinaus“ wurde nicht gebildet, da im Korpus keine solchen Verweise zu finden sind. Ähnliches gilt für Kategorien wie Strasse oder Quartier. Die Grösse Quartier ist in Stans durch eine Tafel vertreten. Ob die areal kleineren Kategorien wie Quartier oder Strasse in grösseren Städten eine bedeutendere Rolle spielen, ist eine spannende Frage, die an dieser Stelle offenbleiben muss.

22 Als Zeichen gelten hier die einzelnen Zeichenträger wie Schilder, Plakate, Tafeln etc.
} 


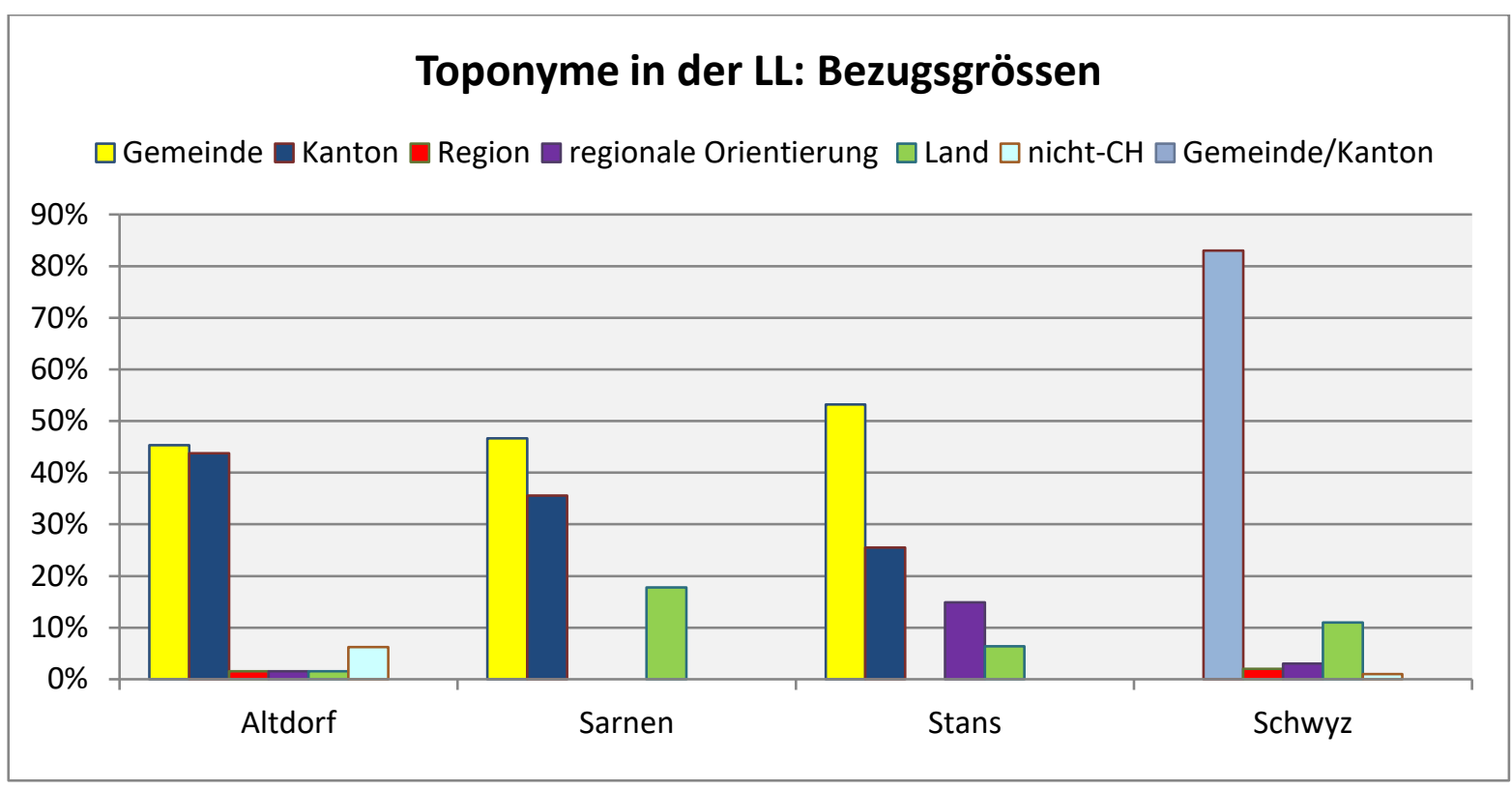

Grafik 2: Verhältnisse der genannten topographischen Grössen, N=256

Wie die Grafik zeigt, überwiegt die Bedeutung der Grössen „Gemeinde“ und „Kanton“ mit grossem Abstand, wobei die Grösse „Gemeinde“ von besonderer Wichtigkeit ist.

Überraschend wenig Bedeutung kommt hingegen der Grösse „Region“ zu. Sie wird in insgesamt 3 Zeichen genannt, diese stehen in den beiden grösseren Ortschaften Altdorf und Schwyz. Die Nennungen sind „Innerschweiz“ oder „Zentralschweiz“, die Bezeichnung „Urschweiz" kommt nicht vor. Dass dennoch engere Beziehungen unter den untersuchten Kantonen bestehen, zeigt der nähere Blick auf die Nennungen zur Kategorie „,regionale Orientierung“. Dabei findet sich nicht nur die zu erwartende Kombination „Ob- und Nidwalden“ (Schaufenster „Natur Umwelt“) bzw. „Stans und Sarnen“ (Schaufenster „Papeterie Spichtig“, beide in Stans). Dies ist eine Kombination, die auf die Zusammenführung von Ob- und Nidwalden zu Unterwalden hinweist (als Nennung ebenfalls einmal vertreten an der Tafel vor der Geschäftsstelle des Schweizerischen Roten Kreuzes in Stans). Die beiden anderen Gemeinden, Altdorf und Schwyz, werden als Niederlassungen am Schaufenster der „Schreinerei W. Bucher" in Schwyz aufgeführt. Diese beiden Kombinationen sind nicht zuletzt auch aufgrund der geographischen Nähe von Ob- und Nidwalden einerseits und Uri und Schwyz andererseits naheliegend. Das Sportgeschäft „3Sixty“ in Schwyz jedoch lässt seine Niederlassungen in Stans und Schwyz auf dem grossen Transparent vor dem Geschäft aufführen, während sich die Computerfirma „Comed AG“ in Stans mit „Altdorf, Schwyz, Stans“ anschreibt. Diese Belege zeigen, dass die Geschäftsverbindungen innerhalb der gesamten Region durchaus lebhaft sind. Umso bemerkenswerter ist der Umstand, dass die Region als Raumkonzept keinen Niederschlag in der LL findet.

Aufschlussreich ist der Blick auf die Nennungen in der Kategorie „Land“. Einige Zeichen verweisen einfach einsprachig auf die Schweiz wie z. B. „Schweizerischer Studentenverein“ (Tafel in Schwyz) oder „Wanderland Schweiz“ (mit regionaler Wanderwegkarte an jedem Bahnhof). Andere Zeichen repräsentieren das Land in seiner offiziellen Viersprachigkeit, z. B. die Tafel zum „Forum der Schweizer Geschichte“ in Schwyz mit dem Namen in Deutsch, Französisch, Italienisch und Rätoromanisch. Eine andere Dimension hat die Verbin- 
dung zwischen Kantonsnamen und der Bezeichnung „Eidgenossenschaft“, wie auf mehreren Tafeln der touristischen Rundgänge zu finden (z. B. der Verweis zum Rathaus in Schwyz bzw. zum „Haus Lusser“ in Altdorf, die jeweils unter dem Schutz „des Kantons Schwyz und der Schweizerischen Eidgenossenschaft" bzw. „des Kantons Uri und der Eidgenossenschaft“ stehen). Hier wird die Existenz der beiden konzeptuellen Grössen „Kanton“ und „Land“ sichtbar. Gleichzeitig verbindet sich mit der Bezeichnung „Eidgenossenschaft" sicherlich ein anderes mentales Raummodell als mit einem Ausdruck wie „Wanderland Schweiz“. Interessant sind jene Anschriften, auf denen der Landesname auf Englisch steht. Dies ist z. B. der Fall auf mehreren Tafeln rund um die Firma Felchlin in Schwyz, die sich konstant als „Felchlin, Switzerland“ anschreibt. Ähnlich verhält es sich mit der Firma „Studio Rueg, Switzerland“ oder mit der Firma „Swisskeramik“, beide in Sarnen. Die vorgefertigten schwarzen Tafeln vor dem „Chäs Ladä“ in Schwyz tragen über der freien, zur Beschriftung bestimmten Fläche das Logo „Le Gruyère AOP, Switzerland“. Der Landesname auf Englisch suggeriert eine ganz andere Perspektive, einen Blick auf die Schweiz von aussen, aus der Sicht einer globalisierten Welt. Die genannten Firmen und Produkte stellen sich als Mitspieler in einem globalen Markt dar. Und auch hier ist das dahinterstehende mentale Raummodell ein ganz anderes. Nicht eine landesumspannende Verbindung wie beim „Schweizerischen Studentenverein“", die sozusagen die grösstmögliche Ausdehnung innerhalb der Landesgrenzen suggeriert, sondern ein Land neben anderen auf der Erdkugel steht hinter der Bezeichnung „Switzerland“.

\section{Heraldische Zeichen und Ortsloyalität}

Der Schweizer Föderalismus basiert u. a. auf der politischen Autonomie der Kantone, die nicht zuletzt auch darin zum Ausdruck kommt, dass jeder Kanton ein eigenes Wappen besitzt. Aber auch die Gemeinden verfügen über eigene Wappen. Diese heraldischen Zeichen haben hohen Wiedererkennungswert und dienen als Symbole lokaler Identität. Sie lassen sich auch ohne sprachliche Zugabe als Verweis auf das Land, auf den Kanton oder auf die Gemeinde einsetzen. Insbesondere vor dem Nationalfeiertag werden Strassen und Häuser mit Fahnen geschmückt, aber auch viele Schaufenster erhalten Dekorationen, die auf das Fest hinweisen. Die Fahnen sind ausserordentlich zahlreich und hängen über Plätzen und an Gebäuden in unterschiedlicher Zusammensetzung. Die Verknüpfung Gemeinde, Kanton, Land ist eine mögliche und häufige Variante (s. Abb. 4). Ohne hier ins Detail zu gehen, sei nur angemerkt, dass die Fahnen in den vier untersuchten Gemeinden in vielen verschiedenen Kombinationen zu sehen waren, wobei sie meist die Vielfalt der Schweiz abbildeten. In keiner der untersuchten Gemeinden jedoch fand sich die Kombination Uri, Schwyz, Ob- und Nidwalden. Die „Urschweiz" scheint also auch in diesem Kontext keine relevante Grösse darzustellen.

Ortsloyalität kommt hingegen sehr häufig zum Ausdruck. Dies ist exemplarisch an der Aufmachung verschiedener Bäckereien abzulesen. Wie Abb. 3 zeigt, werden auf das Fest hin Produkte angeboten, die in der Verpackungsverzierung und/oder im Namen auf eine räumliche Kategorie Bezug nehmen. Hier scheint die Kategorie „Kanton“ einen Vorrang zu haben. Der Verweis erfolgt entweder durch explizite Benennung („Obwaldner Senne-Chäpli“, „Uristierli“, „Nidwaldner Lebkuchen“) oder durch die blosse Darstellung des Wappens (hierfür eignet sich der Stier, das Wappentier von Uri, besonders gut). Eine Bäckerei in Stans wiederum führt ein Produkt mit der schlichten Bezeichnung „Wappen“ im Sortiment. Der nähere 
Blick verrät, dass es sich hierbei um Schokoladestückchen handelt, die jeweils mit dem Wappen von Stans und (darunter!) dem Wappen der Schweiz verziert sind.

Beim Blick auf derartige Zeichen kommt erneut die Frage nach ihrer Indexikalität auf. Im Aufsatz zu den „Sprachlichen Landschaften“ übt Auer (2010: 276) Grundsatzkritik an der bisherigen Forschung, die meist die Sprache im öffentlichen Raum nur anhand ihrer Sichtbarkeit in einem zusammenhängenden Phänomenbereich fasst. Dabei, argumentiert Auer, lassen sich mehrere semiotische Typen unterscheiden. Ein Kriterium ist dabei die Indexikalität der Zeichen. Auer (2010: 276) unterscheidet zwischen indexikalischen Zeichen, die „nur an und durch den Ort interpretierbar sind, an dem sie sich befinden" und nicht-indexikalischen Zeichen, deren Bedeutung ,durch Veränderungen in der Platzierung hingegen nicht in derselben Weise berührt [wird] wie die indexikalischer“ Zeichen (Auer 2010: 278). Letztere „können verstellt, abmontiert, in ihrer Richtung verändert werden, ohne dass sie ihre Funktion verlieren“" (Auer 2010: 278). Vordergründig können die Produkte in den Bäckereischaufenstern als nicht-indexikalische Zeichen klassifiziert werden. Hier sind Name oder Wappen auf der Verpackung an jedem beliebigen Ort interpretierbar. Wie die Bilder zeigen, sind die Produkte im Schaufenster jedoch Teil eines zusammengehörenden Dekorationsarrangements. Dieses bezieht sich jeweils im engeren Sinne auf das Geschäft dahinter, im weiteren Sinne auf die Lokalität, in der sich das Geschäft befindet. Diese Bedeutungslinie (Produkt> ZeichenEnsemble $>$ Schaufenster $>$ Geschäft $>$ Lokalität) lässt sich an keinem anderen Ort erschliessen als an jenem, an dem die Zeichen platziert sind. So kommt hier die Frage auf, ob die einzelnen Dekorationsteile im Hinblick auf ihre Zusammenstellung als Dekorationsarrangement eine zweite Bedeutung erlangen, in diesem Sinne also zugleich indexikalisch und nichtindexikalisch sein können oder ob das gesamte Zeichenensemble ${ }^{23}$ diese Bedeutung transportiert und als ein eigenes, indexikalisches Zeichen gelten kann, während seine einzelnen Bestandteile nicht-indexikalisch sind? Möglicherweise wäre zu unterscheiden zwischen verschiedenen Ausprägungen oder Stufen der Indexikalität. So könnte vielleicht von einer primären Indexikalität ausgegangen werden, die sich daraus ergibt, dass ein Zeichen nur an einem bestimmten Ort interpretierbar ist im Gegensatz zu solchen Zeichen, die unabhängig vom Ort interpretierbar sind. Eine sekundäre Indexikalität ergibt sich jedoch auch bei den primär nicht-indexikalischen Zeichen dadurch, dass sie dennoch an einem bestimmten Ort angebracht sind und durch die Wechselwirkungen mit den anderen Zeichen in der näheren Umgebung weitere Bedeutungsaspekte aktiviert werden. Solche Fragen können an dieser Stelle nicht abschliessend beantwortet werden, doch scheint mir die Weiterführung der Forschungsdiskussion über die Typologie der Zeichen ein dringendes Desiderat der LL-Forschung zu sein. Es lässt sich auf jeden Fall festhalten, dass beim Platzieren von Produkten wie der „Uristierli“ oder der „Nidwaldner Lebkuchen“" nebst Wappen etc. in der Lokalität eines Geschäfts indexikalische Bedeutungsverhältnisse vorhanden sind, wobei diese ähnlich wie im Falle der dialektalen Anschriften auf mentale Raummodelle verweisen. ${ }^{24}$

23 Auer (2010: 286) definiert ein Ensemble als ,,mehrere Schilder/Inschriften/Zettel/Aufkleber, die in unmittelbarer Nähe zueinanderstehen, also mit einem Blick wahrgenommen werden können, und die sich inhaltlich aufeinander beziehen“. Die semiotische Ausrichtung der gesamten Typologie lässt sicherlich auch andere Zeichentypen wie Bilder oder Figuren zu, wie sie in den Schaufenstern stehen.

24 Besonders deutlich, da doppelt vorhanden, ist dieser indexikalische Verweis bei einem Produkt wie die „Obwaldner Senne-Chäppli“, das mehrfach, sowohl ausdrucksseitig als auch inhaltsseitig Zugehörigkeit markiert.

ISSN 1615-3014 


\section{Wilhelm Tell in der LL: der Bezug zur Urschweiz}

Während die „Urschweiz“ als räumliche Grösse in der LL der untersuchten Gemeinden nirgends Niederschlag findet, ist der Gründungsmythos der Schweiz, der ebenfalls hinter der konzeptuellen Grösse „Urschweiz“ steht, durchaus greifbar. In erster Linie wird er durch die Figur von Wilhelm Tell repräsentiert. Altdorf als Hauptort von Uri, der Heimat Tells, sieht sich hier in einer besonders privilegierten Position. Nicht nur ragt im Ortszentrum der mächtige Turm mit dem „Tell-Denkmal“ auf, dem Wahrzeichen Altdorfs. In der Stadt gibt es auch eine „Tellgasse“, an der Busstation „Tell-Denkmal“ hält der „Tell-Bus“ und im öffentlichen Verkehr der Region kann man, wie einem Plakat zu entnehmen ist, mit dem „Tell-Pass“ fahren. Das Gebäude des „Theaters Uri“" nennt sich „Tellspielhaus Altdorf“. Ein weiteres Plakat wirbt für ein Festival in der Region mit dem Slogan „Tell trifft Wagner“. Die reizvolle Zweideutigkeit des Slogans wird getilgt durch die Ergänzung „Begegnungen am Vierwaldstättersee" (auch hier also eine unmittelbare Verknüpfung zu einem Raummodell anhand eines Toponyms). In einem Schaufenster wirbt der Immobilienmakler Toni Bär mit dem Slogan „Treffsicher wie Tell“. Im „Boutique Hotel Reise“ ist man gemäss Anschrift an der Tür „Zuhause bei Wilhelm Tell“, in der Bäckerei kann man „Tells Lieblingsbrot" kaufen und es gibt ein Restaurant/Pizzeria „Wilhelm Tell“. In Stans findet sich ebenfalls ein Restaurant „Wilhelm Tell“, dort kann man einen „Tell-Spiess“ oder „Tell's Salatteller“ bestellen. ${ }^{25}$ In Schwyz ist die Pensionskasse „Pro Tellco“ (mit Tell-Bild im Logo) angeschrieben. Amüsant ist das Logo der Metzgerei Felder AG in Schwyz (Abb. 5): Die Anschrift „Origine Swiss Tell“ enthält Code-mixing, das sowohl den französischsprachigen Landesteil als auch die globale Gemeinschaft zu berücksichtigen versucht, wobei auch inhaltlich Doppeldeutigkeit vorhanden ist: Ist hier der Ursprung der Schweiz gemeint, der irgendetwas mit Tell, aber nichts mit dem Fleisch zu tun haben kann, oder der schweizerische Ursprung des angebotenen Fleisches, der jedoch kaum etwas mit Tell zu tun haben könnte? Dazwischen ragt eine stilisierte Hand mit zum Eid gestreckten Fingern, wie aus den Bildern zum Rütli-Schwur bekannt. Dass weder Tell noch der Rütli-Schwur historisch belegt sind, scheint hier genauso wenig eine Rolle zu spielen, wie auch der Umstand, dass die Figur Tell bei der Gründung der Eidgenossenschaft, wie sie im Mythos dargestellt wird, gar nicht dabei war.

\subsection{Zeichen, die kulturelle Hybridität zum Ausdruck bringen}

Wie die bisherigen Ausführungen gezeigt haben, finden sich in der LL der untersuchten Gemeinden viele Zeichen, die Ortsloyalität und Heimatverbundenheit zum Ausdruck bringen. Es wäre jedoch ein Trugschluss, dies damit zu erklären, dass hier entlegene, rückständige Gemeinden in einer Bergregion im Fokus stünden, die von Migration und Globalisierung unberührt geblieben seien. In der LL der vier Gemeinden kommen verschiedenen Typen kultureller Hybridität zum Ausdruck, von den Ausdrucksformen einer primären kulturellen Hybridität, wie sie Migrantinnen und Migranten selbst auszeichnet, über Ausdrucksformen einer sekundären kulturellen Hybridität, die als Antwort auf Globalisierung und Migration von den Mitgliedern der autochthonen Gesellschaft produziert werden, bis hin zu den Ausdrucksformen von De-Hybridisierung, wie sie durch den Eintritt von Ausdrucksformen primärer oder 
sekundärer kultureller Hybridität in das Repertoire der autochthonen Kultur bei Ausblendung der ethnischen Dimension entsteht (hierzu im Detail Petkova 2014). Exemplarisch erwähnt sei hier die in Holzrahmen gefasste Ankündigung im Menüfenster eines Restaurants (Abb. 6). Das Restaurant befindet sich im Zentrum von Schwyz, in einem alten Haus mit Butzenfenstern, der Name „Rats-Keller“ ist über der Eingangstür in gebrochener Schrift an die Wand gemalt. Die Ankündigung lautet: „Liebe Schwyzerinnen und Schwyzer, liebe auswärtige Gäste, Sie dürfen gespannt sein... Neueröffnung Mitte August 2014, Spezialitäten aus ThailandVietnam-China. Wir freuen uns auf Sie! Familie Vu \& Ratskeller Team“. In dieser Ankündigung wird auf mehrere Räume verwiesen: Zunächst wird die Anwohnerschaft von Schwyz angesprochen („Liebe Schwyzerinnen und Schwyzer“), danach dehnt sich der Radius auf eine unbestimmte Grösse aus, die mit dem Adjektiv ,auswärtig“ dennoch räumlichen Bezug auf Schwyz nimmt (,liebe auswärtige Gäste“). ${ }^{26}$ Das Speiseprogramm des Restaurants stützt sich danach auf die Grösse „Land“ („Spezialitäten aus Thailand-Vietnam-China“). Dies dürfte kein Zufall sein. Denn die Länderküchen, die hier genannt werden, erhalten häufig die allgemeine Bezeichnung ,,asiatische Küche“. Möglicherweise soll die Präzision besondere Authentizität suggerieren. Möglicherweise aber evozieren die Grösse „Gemeinde“ aus der Vor-OrtPerspektive und die Grösse „Land“ aus der Perspektive der überkontinentalen Distanz die maximale Annäherung an die Vorstellung von Nähe und Vertrautheit. Sie sollen also als Akte von place-making das mentale Raummodell eines place (im Gegensatz zum unspezifischen space) evozieren, um die zukünftigen Gäste anzuziehen. Die Verknüpfung des altehrwürdigen Gebäudes mit dem Speiseprogramm des Restaurants, die Verknüpfung der Namen „Familie

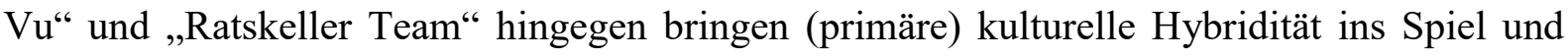
machen eine weitere Spannungslinie sichtbar, die Lokalität, mentale Raummodelle und raumbezogenes Handeln miteinander verbindet. ${ }^{27}$

\section{$7 \quad$ Fazit}

In der LL der Gemeinden Altdorf, Schwyz, Sarnen und Stans wird die mentale Grösse „Raum“ als Bezugsgrösse für Zugehörigkeits- und Identitätsempfinden auf unterschiedliche Weise sichtbar. Zum einen wird in vielen Anschriften Dialekt eingesetzt. Solche Anschriften weisen viele sprachliche Merkmale mit "third-order indexicality" auf, Merkmale, die gemäss Befragungen als besonders typisch und charakteristisch einerseits für den Grossraum „Deutschschweiz“, andererseits für die „Kleinregion“ gelten. Es lassen sich also zwei entsprechende mentale Raummodelle erkennen, die durch das raumbezogene sprachliche Handeln ausdrucksseitig evoziert werden.

\footnotetext{
26 In diesem Kontext darf mit einiger Sicherheit angenommen werden, dass sich die Bezeichnung Schwyz auf die Gemeinde bezieht: Der Ausdruck „liebe Schwyzerinnen und Schwyzer“ ist wohl kaum an die ganze Kantonsbevölkerung adressiert.

27 Zur Kritik daran, dass die Relevanz der Mobilität von der Forschung zu Sprache und Raum häufig ausgeblendet wird, cf. Auer (2013: 6-8).
}

ISSN 1615-3014 


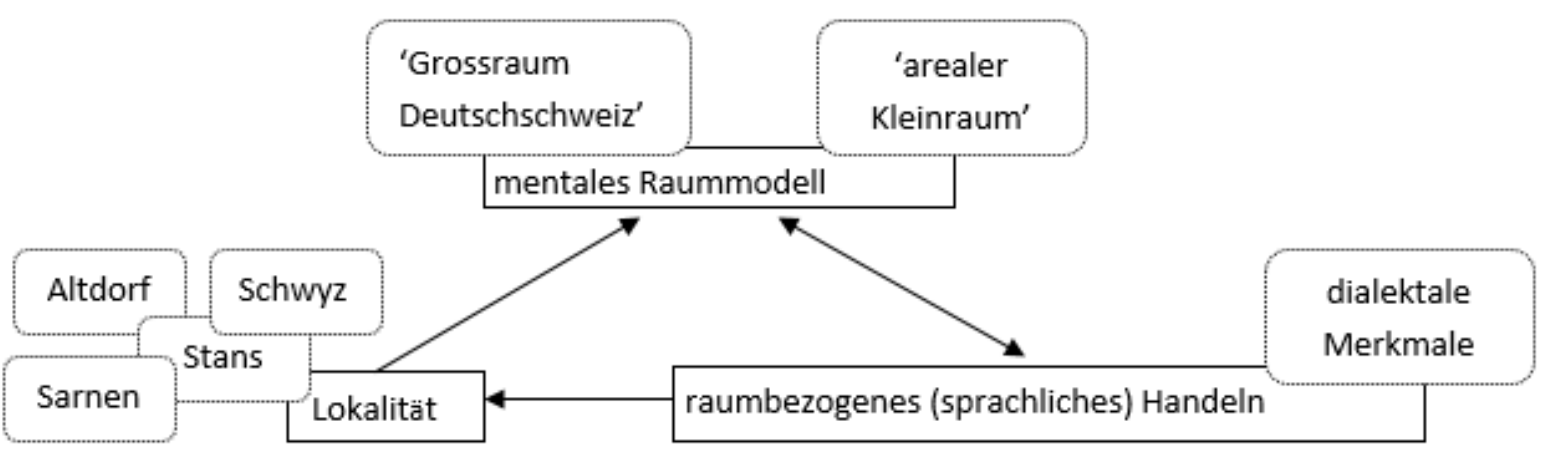

Grafik 3: Ausdrucksseitig, durch den Dialekt vermittelte mentale Raummodelle

Besonders häufig finden sich in der LL der vier Gemeinden toponymische Verweise, die Zugehörigkeit zu einer bestimmten räumlichen Grösse zum Ausdruck bringen. Aber auch einige bildliche Zeichen, insbesondere Wappen oder Fahnen, haben räumlichen Bezug. Mit Abstand am häufigsten vertreten sind hier die Grössen „Gemeinde“ und „Kanton“, wobei die Grösse „Gemeinde“ einen Vorrang hat. „Gemeinde“ und „Kanton“ sind im Vergleich zu Grössen wie „Region“ oder „Land“ die flächenmässig kleineren Grössen. Dies sind zugleich die Grössen, die mit der Auffassung von place in Zusammenhang stehen. Ihr materialisiertes Einschreiben in die LL ist zugleich ein Ausdruck von place-making. Denn wenn place das Vertraute, Nahestehende, Identitätsstiftende repräsentieren soll, so kann dies in erster Linie die nähere Umgebung sein. ${ }^{28}$

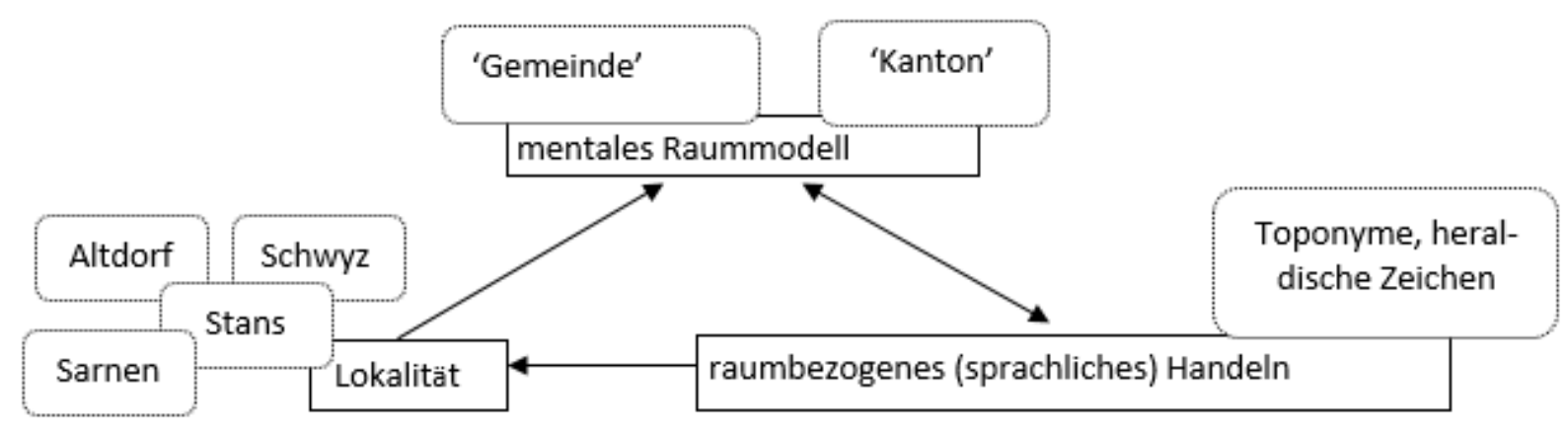

Grafik 4: Inhaltsseitig vermittelte mentale Raummodelle: die meistvertretenen Grössen

Die Grösse „Land“ ist weniger häufig vertreten, doch kommen hier unterschiedliche Bezeichnungen vor. Die verschiedenen Toponyme, die sich auf die gleiche Lokalität beziehen (hier als „die Schweiz im Allgemeinen“ zu begreifen, deren Bestandteil die untersuchten Ortschaften sind), zeugt vom Vorhandensein unterschiedlicher mentaler Repräsentationen. Ob auf die Schweiz als „Eidgenossenschaft“, „Schweiz“, „Wanderland“ oder „Switzerland“ referiert wird, hängt vom jeweils gültigen mentalen Raummodell ab.

28 Cf. auch die Anmerkung von Auer (2013: 17): "Place-making thus works at the level of small neighbourhoods, towns or cities, or smaller regions". 


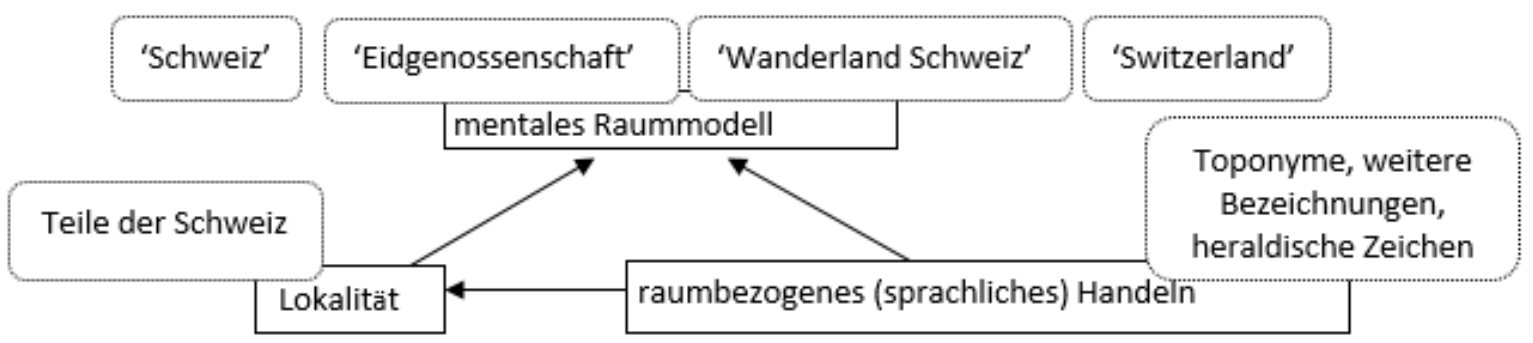

Grafik 5: Inhaltsseitig vermittelte mentale Raummodelle, die auf die Grösse „Land“ Bezug nehmen

Die Urschweiz und der Gründungsmythos der Schweiz werden hauptsächlich durch den TellMythos repräsentiert. $\mathrm{Ob}$ es sich hierbei um ein mentales Raummodell handelt oder ob der Bezug zum Raum nur indirekt vorhanden ist, lässt sich nicht eindeutig bestimmen.

Die linguistic landscapes der untersuchten Gemeinden zeugen davon, dass die gleichen Lokalitäten mit verschiedenen mentalen Raummodellen verknüpft werden, die jeweils das raumbezogene (sprachliche) Handeln prägen. Von Bedeutung ist hier, dass auf beiden Seiten (mentales Raummodell und raumbezogenes Handeln) Vielfalt besteht. Dennoch scheinen die Akte von place-making ein besonderes Gewicht zu haben. Die vertraute, kleinräumige Umgebung kommt an erster Stelle, wenn es darum geht, areale Zugehörigkeit zum Ausdruck zu bringen. Dabei scheint dies in den hier untersuchten kleinen Ortschaften einem Bedürfnis zu entsprechen, wovon die vielen Toponyme insbesondere zu den Grössen „Gemeinde“ und „Kanton“ aber auch die dialektalen Anschriften in der LL zeugen. Wie es sich damit in grösseren Ortschaften und in anderen arealen Kontexten verhält, ist eine Frage, die für weitere Forschung offenbleibt.

\section{Literaturverzeichnis}

Agha, Asif (2003): "The social life of a cultural value". Language \& Communication 23: 231-73.

Ammon, Ulrich (1995): Die deutsche Sprache in Deutschland, Österreich und der Schweiz. Das Problem der nationalen Varietäten. Berlin/New York: de Gruyter.

Ammon, Ulrich et al. (2004): Variantenwörterbuch des Deutschen. Die Standardsprache in Österreich, der Schweiz und Deutschland sowie in Liechtenstein, Luxemburg, Ostbelgien und Südtirol. Berlin/New York: de Gruyter.

Androutsopoulos, Jannis (2014): "Computer-mediated Communication and Linguistic Landscapes”. In: Holmes, Janet/Hazen, Kirk (eds.): Research Methods in Sociolinguistics: A Practical Guide. Chichester, Wiley-Blackwell: 74-90.

Auer, Peter (2004): „Sprache, Grenze, Raum“. Zeitschrift für Sprachwissenschaft 23/2: 149180.

Auer, Peter (2009): "Visible dialect". In: Hovmark, Henrik/Stampe Sletten, Iben/Gudiksen, Asgerd (eds.): I mund og bog. 25 artikler om sprog tilegnet Inge Lise Pedersen på 70årsdagen d. 5. juni 2009. Kopenhagen, Nordisk Forskningsinstitut: 31-46.

Auer, Peter (2010): „Sprachliche Landschaften. Die Strukturierung des öffentlichen Raums durch die geschriebene Sprache“. In: Deppermann, Arnulf/Linke, Angelika (eds.): Sprache intermedial: Stimme und Schrift, Bild und Ton. Berlin/New York, de Gruyter: 271-298. 
Auer, Peter (2013): “The Geography of Language: Steps toward a New Approach". Freiburger Arbeitspapiere zur Germanistischen Linguistik. FRAGL 16. www.portal.unifreiburg.de/sdd/fragl/2013.16. [07.05.17].

Backhaus, Peter (2007): Linguistic Landscape. A Comparative Study of Urban Multilingualism in Tokio. Clevedon: Multilingual Matters.

Ben-Rafael, Eliezer (2009): "A sociological approach to the study of linguistic landscapes". In: Shohamy, Elana/Gorter, Durk (eds.): Linguistic Landscape: Expanding the Scenery. London, Routledge: 40-54.

Ben-Rafael, Eliezer et al. (2006): "Linguistic Landscape as Symbolic Construction of the Public Space: The Case of Israel”. In: Gorter, Durk (ed): Linguistic Landscape. A New Approach to Multilingualism. Clevedon/Buffalo/Toronto, Multilingual Matters: 7-30.

Christen, Helen (2004): „Dialekt-Schreiben oder ,sorry ech hassä Text schribä“““. In: Glaser, Elvira/Ott, Peter/Schwarzenbach, Rudolf (eds.): Alemannisch im Sprachvergleich. Stuttgart, Steiner: $71-85$.

Christen, Helen et al. (2010): Hochdeutsch in aller Munde. Eine empirische Untersuchung zur gesprochenen Standardsprache in der Deutschschweiz. Stuttgart: Steiner.

Cresswell, Tim (2004): Place: a short introduction. Malden/MA: Blackwell.

Gieryn, Thomas F. (2000): “A Space for Place in Sociology”. Annual Review of Sociology 26: 463-496.

Gorter, Durk (2006): "Introduction: The Study of the Linguistic Landscape as a New Approach to Multilingualism”. In: Gorter, Durk (ed): Linguistic Landscape. A New Approach to Multilingualism. Clevedon/Buffalo/Toronto, Multilingual Matters: 1-6.

Harvey, David (1990): "Between Space and Time: Reflections on the Geographical Imagination”. Annals of the Association of American Geographers 80/3: 418-434.

Huebner, Thom (2006): “Bangkok's Linguistic Landscapes: Enviromental Print, Codemixing and Language Change". In: Gorter, Durk (ed.): Linguistic Landscape. A New Approach to Multilingualism. Clevedon/Buffalo/Toronto, Multilingual Matters: 31-51.

Jaworski, Adam/Thurlow, Crispin (2010): "Introducing Semiotic Landscapes". In: Jaworski, Adam/Thurlow, Crispin (eds.): Semiotic Landscapes. Language, Image, Space. London, Continuum: $1-40$.

Johnstone, Barbara (2010): "Language and geographical space". In: Auer, Peter/Schmidt, Jürgen Erich (eds.): Language and Space. An International Handbook of Linguistic Variation. Bd. 1: Theories and Methods. Berlin/New York, de Gruyter: 1-18.

Johnstone, Barbara/Andrus, Jennifer/Danielson, Andrew E. (2006): "Mobility, indexicality, and the enregisterment of 'Pittsburghese'". Journal of English Linguistics 34/2: 77-104.

Landry, Rodrigue/Bourhis, Richard Y. (1997): "Linguistic Landscape and Ethnolinguistic Vitality. An Empirical Study". Journal of Language and Social Psychology 16/1: 23-49.

Lefebvre, Henri (1980): La Présence et l'Absence: Contribution à la Théorie des Représentations. Tournai: Casterman.

Lötscher, Andreas (1989): „Probleme und Problemlösungen bei der Mundartschreibung des Schweizerdeutschen“. Zeitschrift für Dialektologie und Linguistik 56/3: 273-297.

Löw, Martina (2012): Raumsoziologie. 7. Auflage. Frankfurt a. M.: Suhrkamp. 
Petkova, Marina (2014): „Ausdrucksformen des Umgangs mit kultureller Hybridität“. In: Haupt, Sabine (ed.): Tertium datur. Formen und Facetten interkultureller Interferenz. Berlin, LIT: 99-112.

Petkova, Marina (2015): „Zum Verhältnis zwischen Ort, Raum und Sprache: Experimentell elizitierte mentale Modelle hinter zwei Toponymen“. In: Ahner, Helen/Klausmann, Hubert (eds.): Dialekt und Öffentlichkeit. Beiträge zur 18. Arbeitstagung zur alemannischen Dialektologie. Tübingen: Online-Publikation. www.hdl.handle.net/10900/59825. [07.05.17].

Rickheit, Gerd/Weiss, Sabine/Eikmeyer, Hans-Jürgen (2010): Kognitive Linguistik. Theorien, Modelle, Methoden. Tübingen: Francke.

Schiesser, Alexandra (i. V.): „Authentizität durch Sprache. Soziosymbolisch relevante Merkmale als Fundus stilistischer Variation“. In: Christen, Helen et al. (eds.): Räume, Grenzen, Übergänge. Akten des 3. Kongresses der Internationalen Gesellschaft für Dialektologie des Deutschen (IGDD). Stuttgart: Steiner.

Schifferle, Hans-Peter (2012): „Badisches und schweizerisches Alemannisch am mittleren Hochrhein. Gemeinsames und Trennendes“. In: Homburger, Wolfgang et al. (eds.): Grenzüberschreitungen. Der alemannische Raum - Einheit trotz der Grenzen? Ostfildern, Thorbecke: 173-197.

Scollon, Ron/Scollon, Suzie Wong (2003): Discourses in Place. Language in the Material World. London: Routledge.

Shohamy, Elana/Gorter, Durk (eds.) (2009): Linguistic Landscape: Expanding the Scenery. London: Routledge.

Shohamy, Elana/Gorter, Durk (2009): “Introduction”. In: Shohamy, Elana/Gorter, Durk (eds.): Linguistic Landscape: Expanding the Scenery. London, Routledge: 1-10.

Soja, Edward William (1996): Thirdspace: Journeys to Los Angeles and other real-andimagined places. Cambridge/MA: Blackwell.

Spolsky, Bernard (2009): "Prolegomena to a Sociolinguistic Theory of Public Signage". In: Shohamy, Elana/Gorter, Durk (eds.): Linguistic Landscape: Expanding the Scenery. London, Routledge: 25-39.

Spolsky, Bernard/Cooper, Robert Leon (1991): The Languages of Jerusalem. Oxford: Oxford University Press.

Stoeckle, Philipp (2014): Subjektive Dialekträume im alemannischen Dreiländereck. Hildesheim/Zürich/New York: Olms.

Ziegler, Evelyn (2013) „Sehflächen im sozialen Kontext“. In: Cölfen, Hermann/Vosskamp, Patrick (eds.): Unterwegs mit Sprache. Beiträge zur gesellschaftlichen und wissenschaftichen Relevanz der Linguistik. Duisburg, UVRR: 321-344. 


\section{Anhang: Abbildungen}
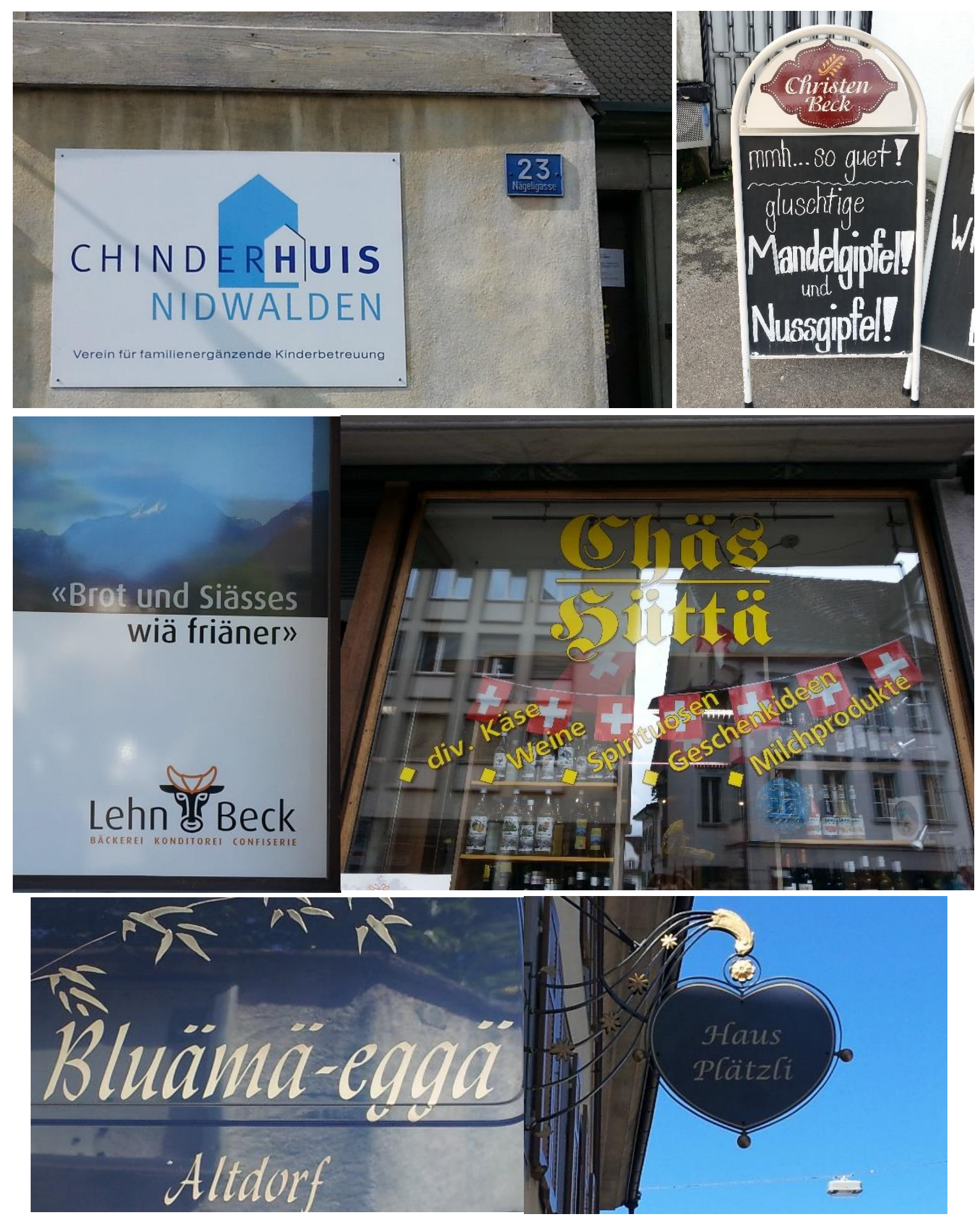

Abbildung 1: Dialektale Anschriften 

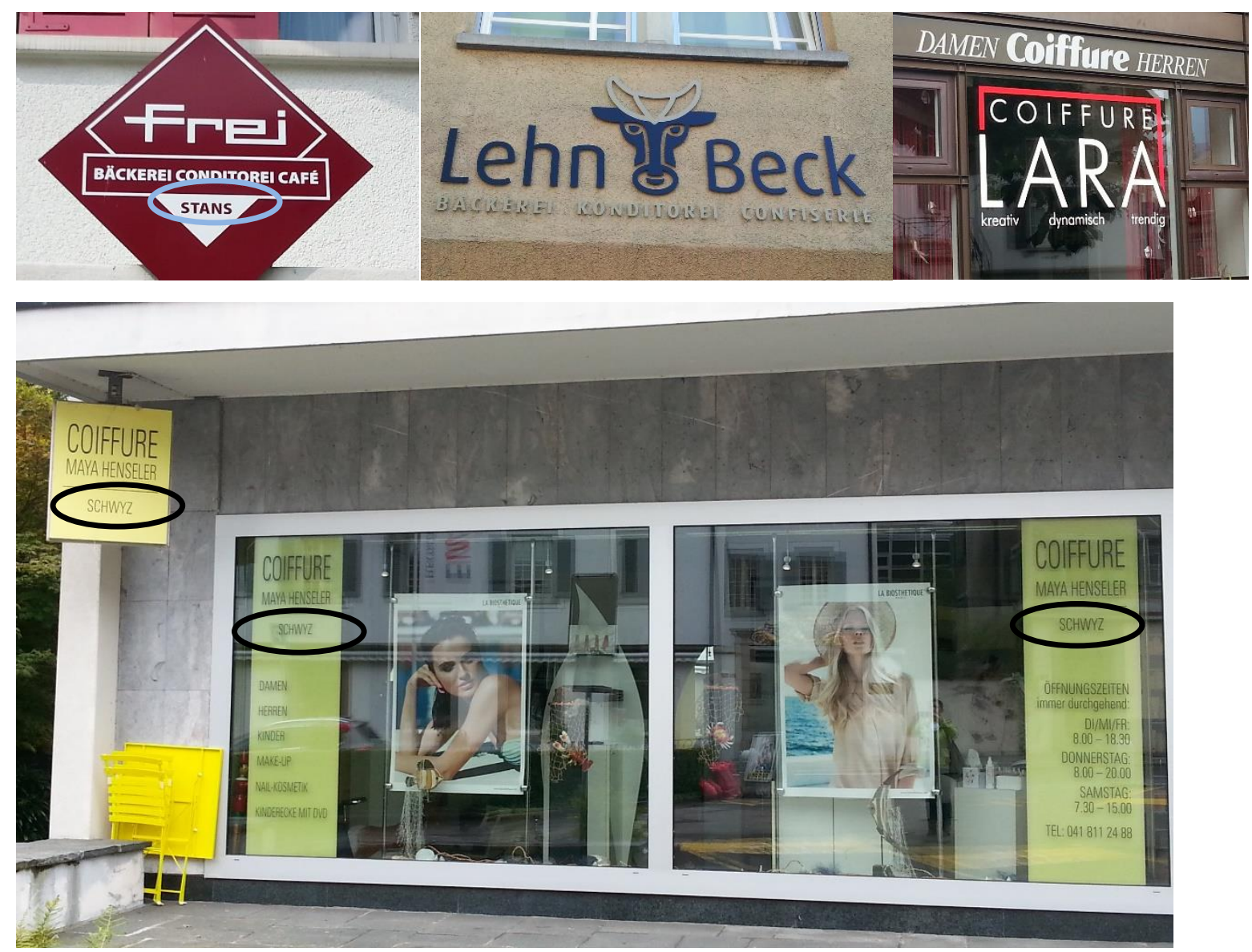

Abbildung 2: Raumzugehörigkeit durch Toponyme und Wappen

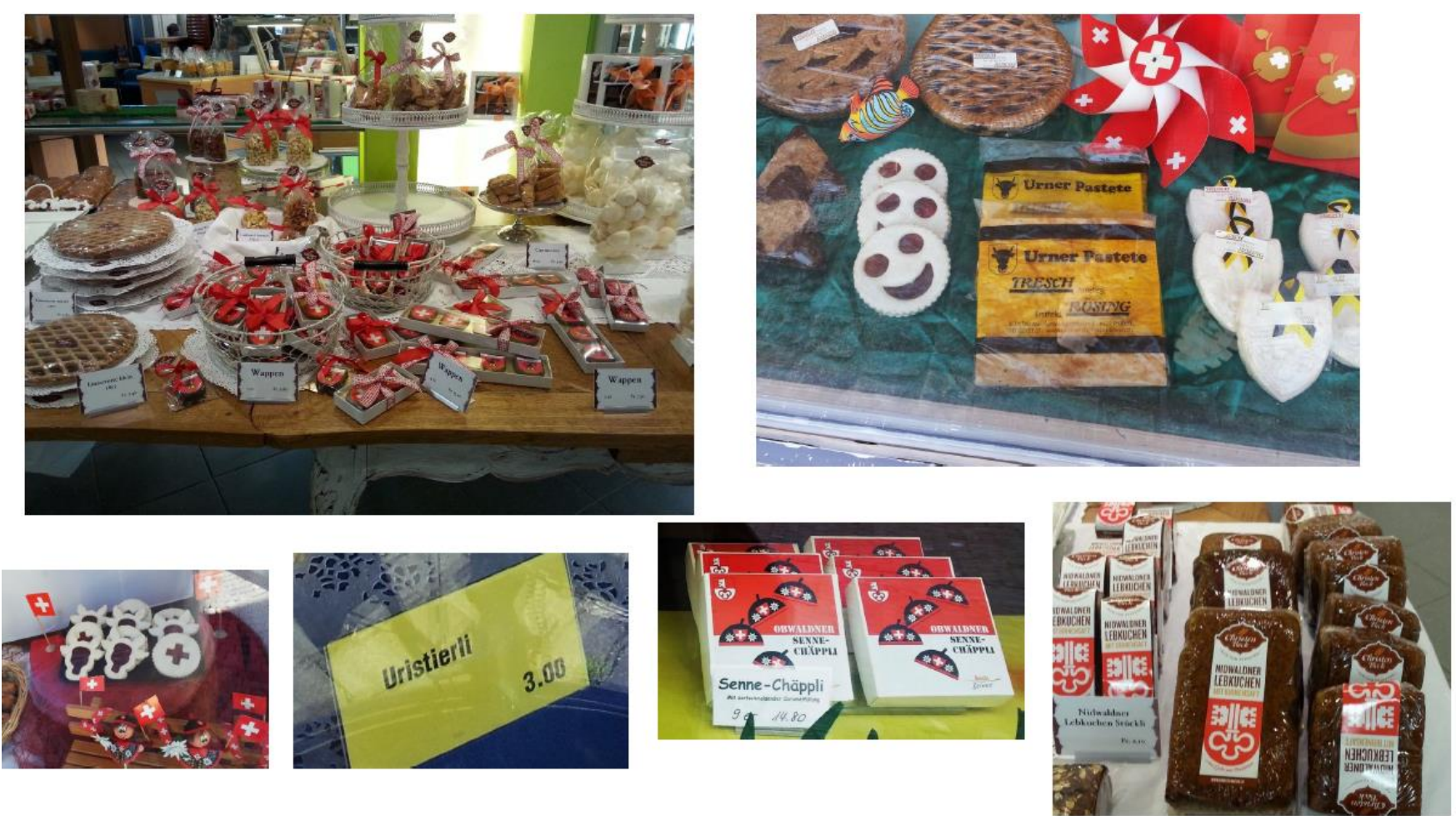

Abbildung 3: Schaufensterdekorationen mit Toponymen, Fahnen und Wappen 


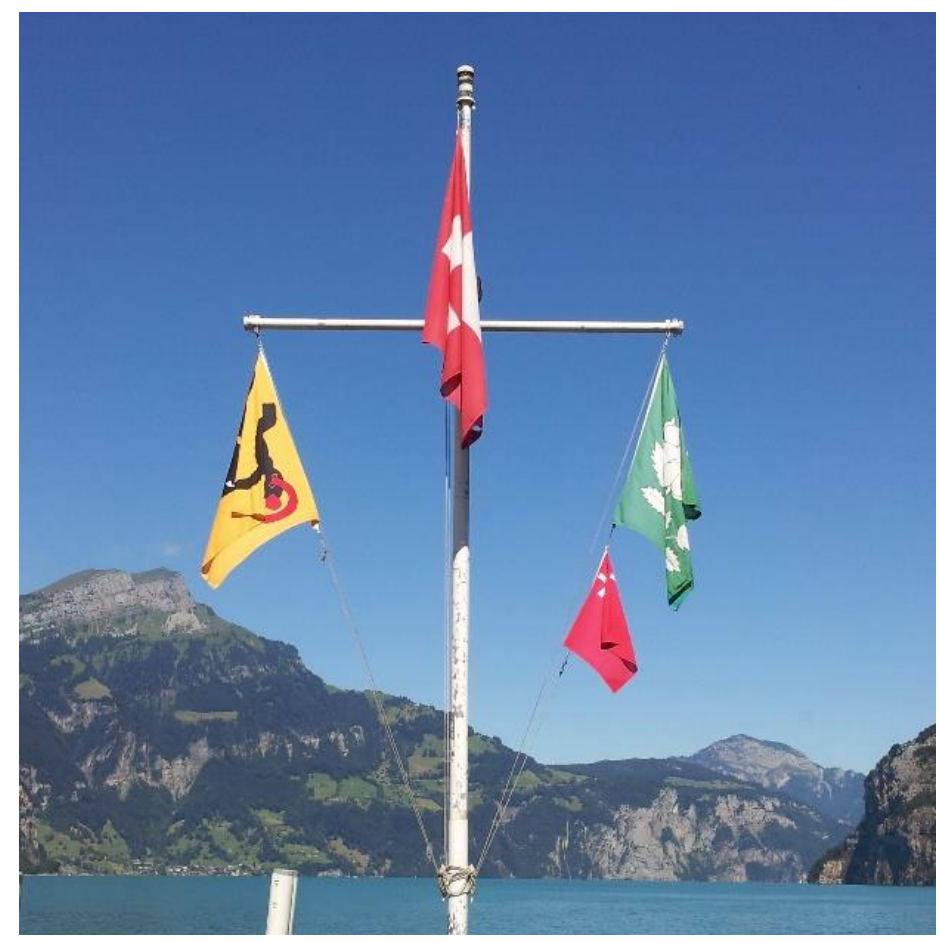

Abbildung 4: Fahnenarrangement an der Schiffsanlegestelle in Flüelen, Uri: Oben die Schweizer Fahne, unten links die Fahne von Uri, unten rechts die Fahne der Gemeinde Flüelen, darunter die Fahne des Kantons Schwyz.

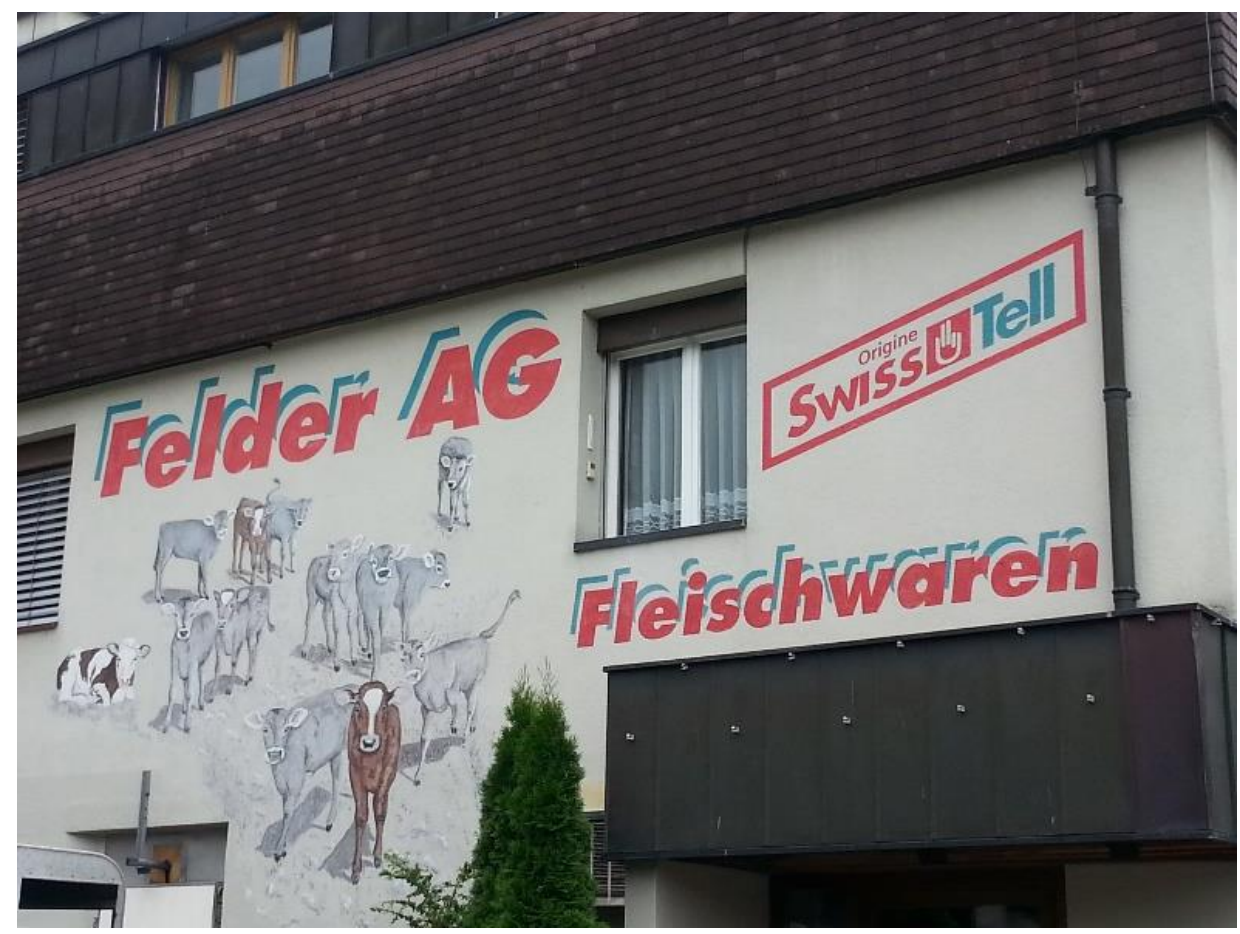

Abbildung 5: Wand der Metzgerei Felder AG, Verweis auf den Gründungsmythos der Schweiz 

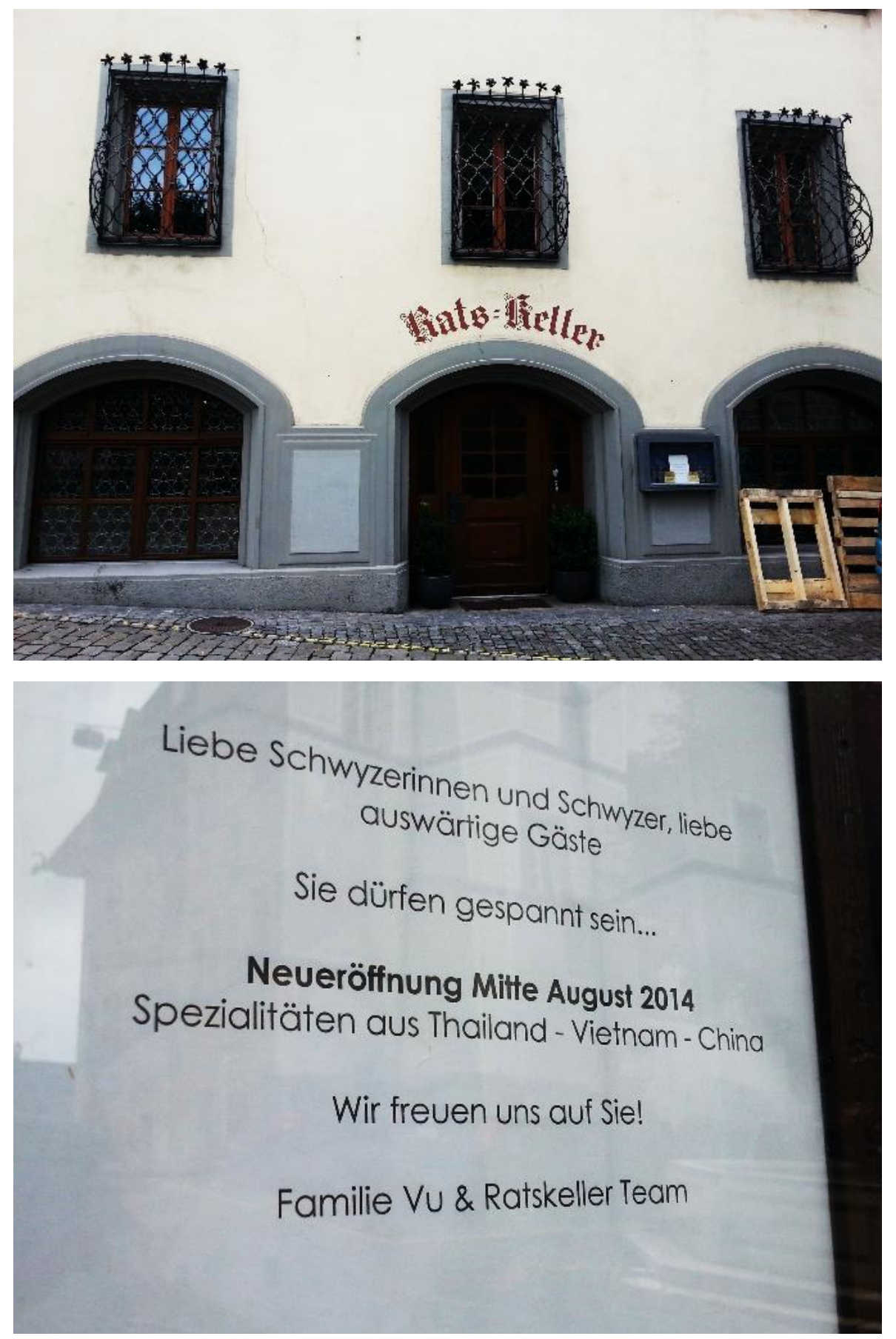

Abbildung 6: Das Restaurant „Rats-Keller“ in Schwyz: Zugehörigkeit und kulturelle Hybridität 